\title{
The compressible mixing layer: an LES study
}

Received: 19 February 2009 / Accepted: 25 August 2009 / Published online: 27 November 2009

(C) The Author(s) 2009. This article is published with open access at Springerlink.com

\begin{abstract}
This article employs LES to simulate temporal mixing layers with Mach numbers ranging from $M_{c}=0.3$ to $M_{c}=1.2$. A form of approximate deconvolution together with a dynamic Smagorinsky subgrid model are employed as subgrid models. A large computational domain is used along with relatively good resolution. The LES results regarding growth rate, turbulence levels, turbulence anisotropy, and pressure-strain correlation show excellent agreement with those available from previous experimental and DNS results of the same flow configuration, underlining the effectiveness and accuracy of properly conducted LES. Coherent structures during the transitional stage change from spanwise aligned rollers to streamwise-aligned thinner vortices at high Mach number. In the quasi-self-similar turbulent stage, the resolved-scale vorticity is more isotropic at higher $M_{c}$, and its vertical correlation length scale is smaller. The ratio of the vertical integral length scale of streamwise velocity fluctuation to a characteristic isotropic estimate is found to decrease with increasing $M_{c}$. Thus, compressibility leads to increased spatial decorrelation of turbulence which is one reason for the reduction in pressure-strain correlation with increasing $M_{c}$. The balance of the resolved-scale fluctuating vorticity is examined, and it is observed that the linear production by mean shear becomes less important compared to nonlinear vortex stretching at high $M_{c}$. A spectral decomposition of the pressure fluctuations into low- and intermediate-to-high-wave numbers is performed. The low-wave number part of the pressure field is found not to correlate with the strain field, although it does have a significant contribution to the r.m.s of the fluctuating pressure. As a consequence, the pressure-strain correlation can be analyzed using a simplified Green's function for the Poisson equation as is demonstrated here using the LES data.
\end{abstract}

Keywords Compressible turbulence · Temporal mixing layer · Pressure-strain correlation . Large-eddy simulation · Enstrophy · Spectral-decomposition

\section{Introduction}

Efficient technology for supersonic aviation, particularly scramjet engines, motivates the interest in compressible turbulent flows. Since compressible flows occur during mixing processes, the transition to turbulence, compression and combustion, simplified flow configurations need to be studied to isolate and distinguish between different compressibility effects, therefore enabling us to get a better understanding. One such simplified flow is the mixing layer between two streams, an important part of any engine. At high speeds, fluid compressibility has been found to strongly affect the behavior of the mixing layer. In order to characterize

H. Foysi $(\bowtie)$

Institute of Aerodynamics, RWTH Aachen University, Wüllnerstr. 5a, 52062 Aachen, Germany

E-mail: h.foysi@aia.rwth-aachen.de

S. Sarkar

Department of Mechanical and Aerospace Engineering, University of California San Diego, 9500 Gilman Dr., La Jolla, CA 92093-0411, USA 
such compressibility effects, the convective Mach number $M_{c}$ has been introduced by Bogdanoff [3], defined as $M_{c}=\left(U_{1}-U_{2}\right) /\left(c_{1}+c_{2}\right)$, with $U_{1}, c_{1}$ and $U_{2}, c_{2}$ denoting the velocity and speed of sound in the high speed stream and low speed stream, respectively.

Numerous investigations, experimentally $[5,7-9,17,29,32,37]$ as well as numerically $[13,14,23,28,34,39$, $40,50,52]$ have tried to understand the influence of compressibility in mixing layers. One of the most important observations is the reduced turbulent shear layer growth rate with increasing convective Mach number $[5,9,17,32,29]$, leading to a stabilization of the flow in the supersonic regime as shown by Sarkar [40]. This reduced thickness of the shear layer has been linked to the decreased turbulent production using an analytical expression by Vreman et al. [50]. In compressible uniform shear flow, the reduced turbulent kinetic energy growth rate has also been shown to be a consequence of reduced turbulent production [40]. This reduction in the turbulent production was shown by DNS studies to be associated with a decrease in the pressure fluctuations, reducing the pressure-strain terms in the turbulent stress balances, in the case of a shear layer by Vreman et al. [50]) and in the case of uniform shear by Sarkar [41]. Later, a study of the annular mixing layer by Freund et al. [13] and a mixing layer by Pantano et al. [28] also found a reduction in pressure fluctuations and pressure-strain terms among other results.

The fluctuating pressure equation has been the subject of analysis to understand the observed compressibility effects. Pantano et al. [28] performed a Green's function analysis without shear for the center of the mixing layer and found that the finite speed of sound in compressible flow causes a time delay for a signal passing a turbulent eddy, causing thereby a decorrelation between adjacent points in this eddy. Recently, Thacker et al. [49] investigated the influence of compressibility on the rapid pressure-strain rate by deriving an exact Green's function for the convected wave equation for pressure fluctuations in homogeneous shear flow. They extended the work done by Papamoschou [30], who used ray theory to investigate the consequence of the wave operator on pressure fluctuations, instead of the usual Poisson equation in incompressible flow, and of Papamoschou et al. [31] who found reduced pressure fluctuation communication in the axial direction in DNS of vortex rings. The Green's function derived by [49] is a combination of parabolic cylinder functions that depend explicitly on the turbulent Mach number $M_{\mathrm{t}}=u_{\mathrm{rms}} / c$ and the gradient Mach number $M_{\mathrm{g}}=\bar{S} l_{\mathrm{I}} / c$, introduced by Cambon et al. [6] and Sarkar [40], with $\bar{S}$ being the mean shear rate and $l_{\mathrm{I}}$ the transverse integral scale. Their results, analyzed in spectral space, indicate, that for higher $M_{\mathrm{g}}$ the stabilizing effect on compressible shear flows increases. Although the Green's function analysis is exact, Thacker et al. [49] introduced simplifications and assumptions, to make the calculation of the pressure-strain correlation analytically tractable. They introduced an isotropic energy spectral density and a form for the anisotropic energy spectrum, together with the assumption of homogeneous shear and an isentropic relation between the pressure and density fluctuations. Contrary to using a convective wave equation for the pressure fluctuations, Foysi et al. [12] and Mahle et al. [23] analyzed a compressible Poisson equation for the pressure fluctuations in supersonic channel flow and in a reacting shear layer with strong heat release. The terms involving time derivatives of the density fluctuations were neglected, as they turned out to be negligible in the calculation of the pressure-strain terms. Such an assumption directly leads to a Green's function solution of the Poisson equation which coincides with the incompressible Green's function solution, as obtained, e.g., by Kim [16]. In view of the results obtained by [49], the fact that the incompressible Green function works for the investigated compressible channel flows in Foysi et al. [12] can be understood by the low $M_{\mathrm{t}}$ and $M_{\mathrm{g}}$.

The structure of the flow is found to become strongly three-dimensional for convective Mach numbers greater than $0.6[8,19]$, whereas for lower Mach numbers similar roll-up and pairing mechanisms as in incompressible flow can be observed [7,18]. Furthermore, linear stability analysis $[34,39,53]$ of temporal and spatial stability problems showed the two-dimensional nature of the most amplified disturbances up to convective Mach numbers of 0.6, whereas for higher Mach number the Kelvin-Helmholtz instability is inhibited (Normand [27]). In addition, the turbulent structures decrease in size with increasing convective Mach number (Lesieur et al. [19] and references therein), demanding better resolution and therefore larger computational resources for higher Mach numbers. Despite the low Reynolds number limitations, direct numerical simulations are capable of providing valuable insight into the processes involved in compressible mixing layers.

Vreman et al. [52] performed a detailed study of various subgrid models in the turbulent mixing layer at a low value of $M_{c}=0.2$ corresponding to quasi-incompressible behavior. In tests at $R e_{\omega 0}=50$ (Reynolds number based on the initial vorticity thickness), a value low enough to be accessible to DNS, the dynamic mixed model was found to give the best overall agreement between the filtered DNS and the LES. In LES at higher Reynolds number, $R e_{\omega 0}=500$ and $R e_{\omega \mathrm{f}}=5000$, the dynamic eddy viscosity model was found to outperform other models with respect to showing self-similar evolution. Vreman et al. [51] considered subgrid 
modeling for compressible terms by performing LES of a mixing layer at low $R e_{\omega 0}=50$, and two values of $M_{c}=0.4$ and 0.6 . They found that, at $M_{c}=0.6$, inclusion of a model for the subgrid dissipation in the energy equation led to a noticeable improvement. The LES study of these authors did not focus on the influence of $M_{c}$ on the flow evolution.

This work revisits the compressible mixing layer to address unresolved issues. It has not been demonstrated that LES has the ability to capture self-similar evolution of the high-speed mixing layer and accurately represent the strong compressibility effects that were briefly reviewed in the preceding sections. We will perform LES for a Reynolds number based on the final vorticity thickness, $R e_{\omega}$, as high as 20,000 and demonstrate again that LES, when properly conducted, has the ability to capture compressibility effects in the mixing layer. The reduction of the pressure fluctuations with increasing $M_{c}$ is not well understood. We will investigate the pressure field using spectral decomposition, a Green's function analysis, and visualization to gain insight. The pressure-strain correlation will also be analyzed. Lastly, the LES database will be examined for compressibility effects on the resolved vorticity field using statistical analysis.

\section{Mathematical formulation}

The present code has the capability to solve for the compressible Navier-Stokes equations using either the total energy equation or the internal energy equation. Here, the latter equation is used since it proved to be more stable in the context of LES. Figure 1 shows the flow configuration, where two streams with different velocities $U_{1}=-U_{2}=\Delta U / 2$ but equal densities are considered. Table 1 shows some reference parameters at the start of the simulation. A large domain size is used to obtain quasi-self-similar evolution, as discussed in Appendix A. The large scales of the flow are well-represented by the chosen domain as demonstrated using two-point correlations in Appendix B. The simulation is not a DNS, since the grid size is much larger than the Kolmogorov scale, for example, $\Delta x_{2}$ varies between $300 \eta$ during the initial transient and $50 \eta$ during the quasi-self-similar stage. Thus, the LES approach is adopted with a subgrid model described later in this

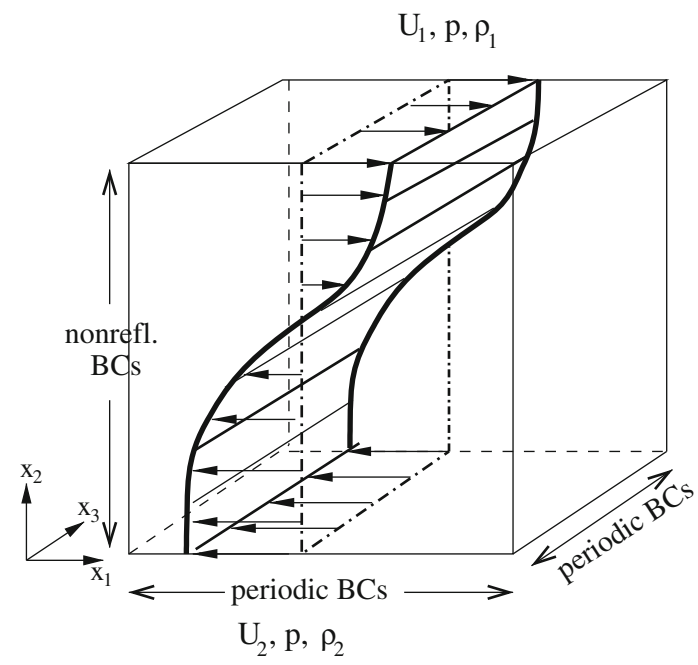

Fig. 1 Sketch of the domain and flow configuration (in this article $U_{2}=-U_{1}, \rho_{2}=\rho_{1}$ )

Table 1 Initial parameters of the simulation

\begin{tabular}{llllllllllll}
\hline Case & $R e$ & $M_{c}$ & $R e_{\omega 0}$ & $\delta_{\theta 0}$ & $\delta_{\omega 0}$ & $N_{1}$ & $N_{2}$ & $N_{3}$ & $\frac{L_{1}}{\delta_{\theta 0}}$ & $\frac{L_{2}}{\delta_{\theta 0}}$ & $\frac{L_{3}}{\delta_{\theta 0}}$ \\
\hline LES03 & 800 & 0.3 & 705 & 0.093 & 0.41 & 768 & 193 & 64 & 1720 & 387 & 172 \\
LES07 & 800 & 0.7 & 705 & 0.093 & 0.41 & 512 & 193 & 140 & 1147 & 344 & 269 \\
LES09 & 800 & 0.9 & 705 & 0.093 & 0.41 & 768 & 193 & 96 & 1720 & 344 & 118 \\
LES10 & 800 & 1.0 & 705 & 0.093 & 0.41 & 512 & 193 & 160 & 1147 & 344 & 172 \\
LES12 & 800 & 1.2 & 705 & 0.093 & 0.41 & 512 & 193 & 160 & 1147 & 344 & 172 \\
\hline
\end{tabular}

$\delta_{\theta 0}$ and $\delta_{\omega 0}$ denote the initial momentum and vorticity thickness, respectively, $R e_{\omega 0}$ the Reynolds number based on the initial vorticity thickness. $L_{i}$ are the domain lengths and $N_{i}$ the corresponding number of grid points 
section. The LES grid is relatively fine in the sense that the energy-containing scales are well-resolved; during the self-similar evolution, there are 20-40 grid points per integral scale in the streamwise direction.

The final value of the Reynolds number $R e_{\omega}$, is as large as 20,000. The Prandtl number is set to 0.7, while the ratio of the specific heats is $\gamma=1$.4. Periodic boundary conditions are used in the streamwise and spanwise direction, whereas non-reflecting boundary conditions [48] together with grid stretching and a sponge layer are used in the normal direction [21]. The initial velocity profile for $u_{1}$ is given as $u_{1}=(\Delta U / 2) \tanh \left(-x_{2} / \delta_{\theta}(0)\right)$. To trigger the transition to turbulence, broadband disturbances are superimposed on the initial velocity profiles. The disturbances are calculated using random fields for the velocity vector potential, $\Psi$, on which a spectrum of the form $\hat{\Psi}_{i} \propto \exp \left(-2\left(k / k_{0}\right)\right)$ is imposed, where $k$ is the wave vector magnitude and $k_{0} / \delta_{\theta 0}=23.66$ the peak wave number. The resultant disturbance field is then restricted to the shear layer by multiplying it with a shape function of the form $\exp \left(-\left(x_{2} /\left(2 \delta_{\theta}(0)\right)\right)^{2}\right)$. The velocity fluctuations are then obtained by taking the curl of the vector potential $\boldsymbol{u}^{\prime}=\nabla \times \boldsymbol{\Psi}$, thus guaranteeing solenoidality. The broadband forcing used here enabled a fast transition to turbulence and limited the emission of acoustic waves initially. This reduction of compressibility transients when using quasi-solenoidal initial perturbations was demonstrated by [10]. here.

The following two sections give an overview of the governing equations and the LES models employed

\subsection{Governing equations}

The Navier-Stokes equations in conservative form for the density, momentum and temperature are filtered using a top-hat filter with filter width $\Delta$, indicating the smallest resolved scale on the LES grid. The filter width $\Delta$ in a given direction was chosen to be the corresponding value of $2 \Delta x_{i}$ (see Vreman et al. [50] and discussion therein). As a consequence, a flow variable $f$ can be decomposed as $f=\bar{f}+f_{\text {sg }}$, with the filtered large-scale part $\bar{f}$ and the unresolved subgrid part $f_{\text {sg. }}$. After Favre [11], a mass weighted filtering operation, $\tilde{f}=\overline{\rho f} / \bar{\rho}$, is used, simplifying the equations considerably. The filtered compressible Navier-Stokes equations used here are:

$$
\begin{gathered}
\frac{\partial \bar{\rho}}{\partial t}+\frac{\partial \bar{\rho} \tilde{u}_{j}}{\partial x_{j}}=0 \\
\frac{\partial \bar{\rho} \tilde{u}_{i}}{\partial t}+\frac{\partial \bar{\rho} \tilde{u}_{i} \tilde{u}_{j}}{\partial x_{j}}+\frac{\partial \bar{p}}{\partial x_{i}}-\frac{\partial \bar{\sigma}_{i j}}{\partial x_{j}}=-\frac{\partial \bar{\rho} \tau_{i j}}{\partial x_{j}} \\
C_{v}\left(\frac{\partial \bar{\rho} \tilde{T}}{\partial t}+\frac{\partial \bar{\rho} \tilde{u}_{j} \tilde{T}}{\partial x_{j}}\right)-\frac{\gamma-1}{\gamma \operatorname{Re}} \bar{\sigma}_{i j} \frac{\partial \tilde{u}_{i}}{\partial x_{j}}+\frac{1}{\operatorname{RePr}} \frac{\partial \bar{q}_{j}}{\partial x_{j}}=-\frac{\partial Q_{j}}{\partial x_{j}}
\end{gathered}
$$

with $\bar{\rho} \tilde{T}=\bar{p}, \gamma M_{\text {ref }}^{2}=p_{\text {ref }}^{2} / \rho_{\text {ref }} U_{\text {ref }}^{2}$ chosen to be unity, and the resolved viscous stress tensor,

$$
\bar{\sigma}_{i j}=2 \frac{\bar{\mu}}{R e}\left(\tilde{S}_{i j}-\frac{1}{3} \delta_{i j} \tilde{S}_{k k}\right), \quad \tilde{S}_{i j}=\frac{1}{2}\left(\frac{\partial \tilde{u}_{i}}{\partial x_{j}}+\frac{\partial \tilde{u}_{j}}{\partial x_{i}}\right),
$$

and the resolved heat flux,

$$
\bar{q}_{j}=-\kappa \frac{\partial \bar{T}}{\partial x_{j}} .
$$

Here, $\delta_{i j}$ is the Kronecker delta and the viscosity $\mu$ is calculated using Sutherland's law. Other subgrid contributions of the molecular diffusivity to the momentum transport equation,

$$
\sigma_{i j}^{\mathrm{sg} 1}=2 \frac{\mu_{\mathrm{sg}}}{\operatorname{Re}}\left(\tilde{S}_{i j}-\frac{1}{3} \delta_{i j} \tilde{S}_{k k}\right) \sigma_{i j}^{\mathrm{sg} 2}=2 \frac{\mu_{\mathrm{sg}}}{\operatorname{Re}}\left(\tilde{S}_{i j}^{\mathrm{sg}}-\frac{1}{3} \delta_{i j} \tilde{S}_{k k}^{s g}\right) \text { and } \sigma_{i j}^{\mathrm{sg} 3}=2 \frac{\bar{\mu}}{\operatorname{Re}}\left(\tilde{S}_{i j}^{\mathrm{sg}}-\frac{1}{3} \delta_{i j} \tilde{S}_{k k}^{\mathrm{sg}}\right),
$$

were found negligible and only that of the subgrid stress tensor,

$$
\tau_{i j}=\widetilde{u_{i} u_{j}}-\tilde{u}_{i} \tilde{u}_{j},
$$

is retained. Similarly, in the temperature equation, the subgrid heat flux

$$
Q_{j}=C_{v}\left(\widetilde{\rho} \widetilde{u_{j} T}-\bar{\rho} \tilde{u}_{j} \tilde{T}\right) .
$$


Table 2 Comparison of grid size of the current LES, normalized with $\delta_{\theta 0}$

\begin{tabular}{lllll}
\hline Case & $\Delta x_{1}$ & $\min . \Delta x_{2}$ & $\max . \Delta x_{2}$ & $\Delta x_{3}$ \\
\hline LES03 & 2.24 & 1.73 & 5.32 & 2.69 \\
LES07 & 2.24 & 1.73 & 3.21 & 1.92 \\
LES09 & 2.24 & 1.73 & 3.21 & 1.23 \\
LES10 & 2.24 & 1.73 & 3.21 & 1.08 \\
LES12 & 2.24 & 1.73 & 3.21 & 1.08 \\
\hline
\end{tabular}

The DNS of [28] has a lower Reynolds number, and grid spacing of 0.67 for all simulations as well as directions

is retained while the subgrid molecular dissipation rate is neglected. The spatial derivatives are calculated using explicit finite difference dispersion relation preserving (DRP) schemes having the summation by parts (SBP) property [15] for the first derivatives. These schemes are of sixth order in the interior and of third order at the boundary. The second derivatives in the viscous terms are calculated using SBP operators of fourth order [25]. Time integration was performed by using a fourth order explicit low dissipation-dispersion Runge-Kutta scheme of Berland et al. [2]. The code was validated by performing DNS of a temporal mixing layer with the same parameters as that of case A3 in Pantano et al. [28] and successfully comparing the statistics.

\subsection{Subgrid modeling}

Table 2 shows the grid spacing of the current LES simulations, as compared to the constant grid spacing of 0.67 in the DNS of Pantano et al. [28]. Although the number of grid points is comparable to the number of grid points in the referred DNS, the grid spacing is much coarser due to the extended domain size. It was found that a large number of grid points was required to obtain smooth statistics, especially in the context of the pressure Poisson equation to be discussed later.

The number of points required in the spanwise direction proved to be crucial, since the transition process changes dramatically with increasing $M_{c}$. This is illustrated in Fig. 2, comparing cases LES03 and LES10 during three different time instants. Spanwise coherent vortices with cross-links can be seen at $M_{c}=0.3$ unlike the thinner streamwise-elongated structures, reminiscent of Lambda vortices at $M_{c}=1.0$. These results, although surely dependent on initial perturbation level and exact form of the spectrum, are consistent with the summary in the book of Lesieur et al. [19] that, above $M_{c}=0.6$, no roll-up and helical pairing occur since the Kelvin-Helmholtz instability is inhibited, the transition process gets three-dimensional and the pressure becomes reconnected into longitudinal tubes. Figure 3 shows isosurfaces of the pressure fluctuations (the value was chosen to be approximately $p_{\text {rms }}$ ) for the same LES cases in the quasi-self-similar stage. Although the differences are less substantial than during transition, again one observes less spanwise coherence and slightly smaller structures for higher $M_{c}$. Overall, it is found that the $M_{c}=1.0$ case requires more grid points and slightly longer domains in the spanwise direction compared to the $M_{c}=0.3$ case to represent the vortical structures. This conclusion was found to hold when an alternate eduction criteria, the $Q$ invariant, was used.

The subgrid model used is a combination of the direct filtering version of the approximate deconvolution model of ADM, originally proposed by Stolz et al. [46], which has been successfully used by Mathew et al. [24] and later Bogey et al. [4] and the compressible variant of the dynamic Smagorinsky model [26]. This combination was chosen due to the observations that reconstruction type models, e.g., scale-similarity model and ADM are able to predict well the subgrid scale tensor structure and its related anisotropic and disequilibrium effects, but lacks the physical contribution of the unresolved fine scales and has less energy transfer $[42,38]$. The subgrid model used here can also be viewed as deconvolution combined with the dynamic Smagorinsky model for regularization.

\subsubsection{Dynamic Smagorinsky}

The stress $\tau_{i j}$ is decomposed into a deviatoric and isotropic part $\tau_{i j}=\tau_{i j}^{\mathrm{D}}+\tau^{\mathrm{I}} \delta_{i j}$, with $\tau^{\mathrm{I}}$ set to zero in the following. The deviatoric part is modeled as [26]

$$
\bar{\rho} \tau_{i j}^{\mathrm{D}}=-2 \bar{\rho} v_{\mathrm{t}}\left(\tilde{S}_{i j}-\frac{1}{3} \tilde{S}_{k k}\right)=-2 \bar{\rho}\left(C_{\mathrm{s}} \Delta\right)^{2}|\tilde{S}|\left(\tilde{S}_{i j}-\frac{1}{3} \tilde{S}_{k k}\right)
$$

with $|\tilde{S}|=\left(2 \tilde{S}_{i j} \tilde{S}_{i j}\right)^{1 / 2}$ and $\left(C_{\mathrm{s}} \Delta\right)^{2}$ determined using the dynamic procedure as 
LES03

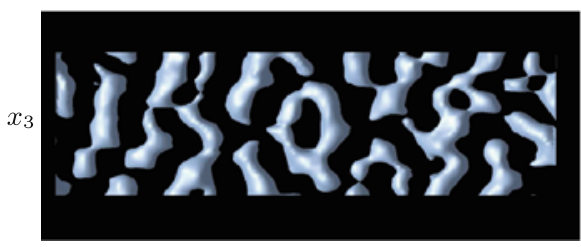

$x_{1}$

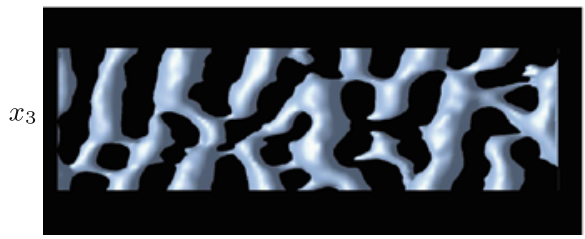

$x_{1}$

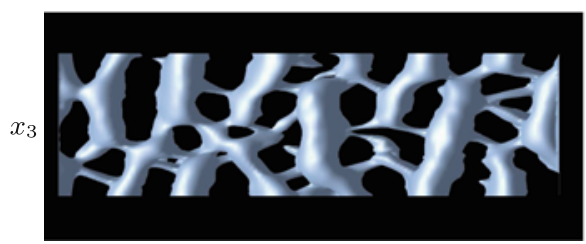

$x_{1}$
LES10

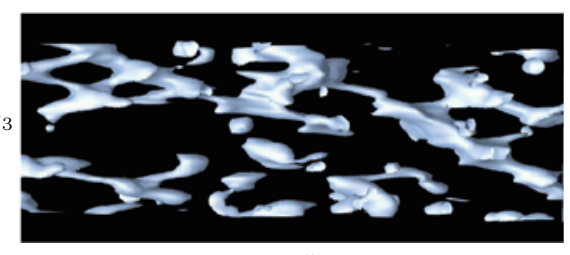

$x_{1}$

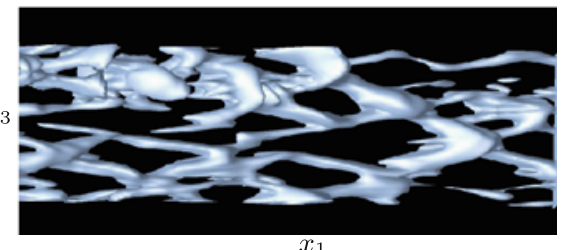

$x_{1}$

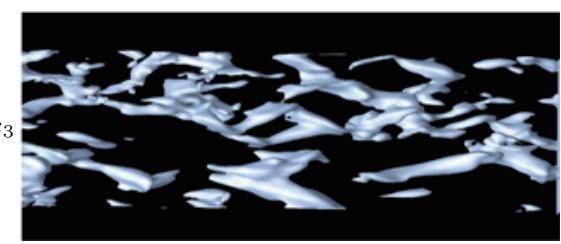

$x_{1}$

Fig. 2 Pressure isosurfaces (viewed from the top for cases LES03 and LES10 at $x_{2}=0$ during three different instants during transition: $t \Delta u / \delta_{\theta}=125$ (top), $t \Delta u / \delta_{\theta}=250$ (middle) and $t \Delta u / \delta_{\theta}=375$ (bottom)

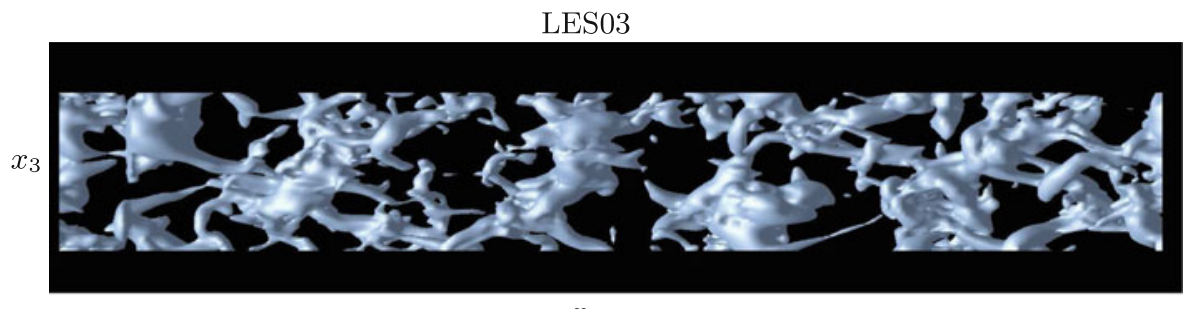

$x_{1}$

LES10

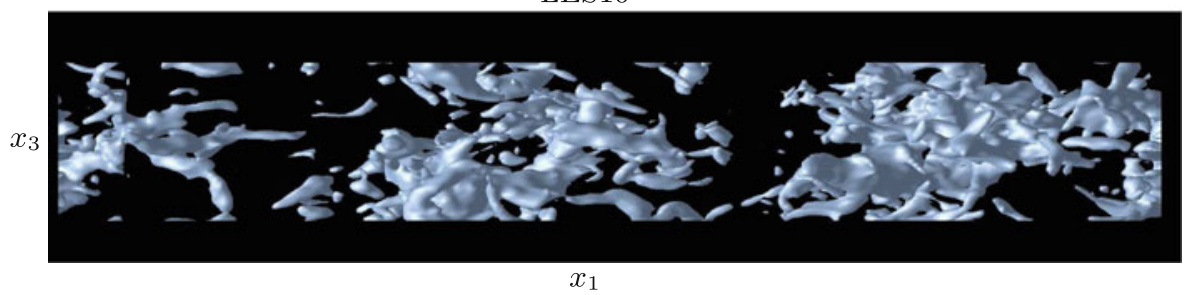

Fig. 3 Pressure isosurfaces for cases LES03 and LES10 at $x_{2}=0$ during the quasi-self-similar stage

$$
\left(C_{\mathrm{s}} \Delta\right)^{2}=\frac{<L_{i j}^{\mathrm{D}} M_{i j}^{\mathrm{D}}>}{<M_{i j}^{\mathrm{D}} M_{i j}^{\mathrm{D}}>},
$$

with

$$
\begin{aligned}
L_{i j}^{\mathrm{D}} & =\widehat{\bar{\rho} \tilde{u}_{i} \tilde{u}_{j}}-\frac{\widehat{\overline{\rho u_{i}}} \widehat{\widehat{\rho u_{j}}}}{\hat{\bar{\rho}}} \\
M_{i j}^{\mathrm{D}} & =2\left[\alpha \hat{\bar{\rho}}|\tilde{\tilde{S}}| \tilde{\tilde{S}}_{i j}^{\mathrm{D}}-\widehat{\bar{\rho}|\tilde{S}| \tilde{S}_{i j}^{\mathrm{D}}}\right]
\end{aligned}
$$


and $\alpha=(\hat{\Delta} / \Delta)^{2}=4$. The test filter operation on $f$ is written as $\hat{f}$ and is an explicit midpoint filter having twice the filter width. The Favre-filtered analogy is $\tilde{\tilde{f}}=\widehat{\overline{\rho f}} / \hat{\tilde{\rho}}$. The subgrid heat flux is modeled as [19]

$$
\left.Q_{j}=C_{\mathrm{v}} \bar{\rho} \widetilde{\left(u_{j} T\right.}-\tilde{u}_{j} \tilde{T}\right)=-C_{\mathrm{v}} \frac{\bar{\rho} v_{\mathrm{t}}}{P r_{\mathrm{t}}} \frac{\partial \tilde{T}}{\partial x_{j}},
$$

with $v_{\mathrm{t}}$ coming from the dynamic Smagorinsky model and the turbulent Prandtl number chosen as $P r_{\mathrm{t}}=0.71$. The simulations proved to be quite insensitive with respect to the value of $P r_{\mathrm{t}}$.

\subsubsection{Approximate deconvolution modeling (ADM)}

ADM [46] models avoid the closure problem, by replacing the fields in the nonlinear terms by their approximate deconvolved counterparts, obtained by applying the approximate deconvolution operator $Q \approx G^{-1}$ to a quantity $f$ to obtain $f^{*}=Q * \bar{f}=Q * G * f$, with the filter operator denoted by $G$. The nonlinear term in the momentum equation $\frac{\partial \bar{\rho} u_{i} u_{j}}{\partial x_{j}}$, for example, is replaced by $\frac{\partial \overline{\rho^{*} u_{i}^{*} u_{j}^{*}}}{\partial x_{j}}$. Mathew et al. [24] show that ADM is equivalent to filtering after each timestep: the filtering operation $G * u$ and the approximate inverse $Q=\sum_{n=0}^{N}(I-G)^{n}[46]$ can be combined into one equivalent filtering step $Q * G * u=: G_{T} * u$. Instead of calculating $Q$ via the series expansion as outlined above, which is quite expensive, an 11-point filter with a transfer function similar to $Q_{\mathrm{T}}$ was designed by [4] and used here in the homogeneous directions. In the vertical direction this approach proved to be too dissipative, due to the applied grid stretching. Therefore, $Q * G$ with $N=4$ was used, applying the filter developed by [47] for non-equidistant grids. Bogey et al. [4] found it sufficient to filter the flow every second timestep. Due to the combination with the dynamic Smagorinsky model, filtering after every 20 timesteps for LES03 was performed to retain numerical stability, whereas for the LES09 and LES12 case filtering was applied after every other timestep, according to $[4,24]$. For the latter cases shocklets, indicated by strong negative dilatation started to form.

\section{Compressibility effects on turbulence statistics}

If LES is to fulfill its promise, compressibility effects seen in DNS and experiments, for example, the reduction of the growth rate, the decrease in the magnitude of the Reynolds stresses, production and pressure-strain correlation should be observed. The cross-stream coordinate will be normalized by either the vorticity thickness, $\delta_{\omega}=\Delta U /\left(d<U>/ \mathrm{d} x_{2}\right)_{\max }$, or the momentum thickness, $\delta_{\theta}$, with the former preferred when previous laboratory or simulation results are given in terms of the vorticity thickness. In the self-similar stage, $\delta_{\omega} \simeq 5 \delta_{\theta}$.

Reynolds averages are denoted by $\langle\cdot\rangle$ and Favre averages by

$$
\langle\phi\rangle^{f}=\frac{\langle\rho \phi\rangle}{\langle\rho\rangle} .
$$

Reynolds averages in the LES simulations are calculated by using plane averaging in the $x_{1}, x_{3}$-directions. Reynolds fluctuating quantities are denoted by $\phi^{\prime}$ and Favre fluctuations as $\phi^{\prime \prime}$ in the following. The turbulent stress tensor is written as (overbar and tilde to indicate filtered quantities are omitted in the following to ease readability, otherwise explicitly stated)

$$
R_{i j}=\frac{\left\langle\rho u_{i}^{\prime} u_{j}^{\prime}\right\rangle}{\langle\rho\rangle}
$$

The turbulent production, resolved dissipation, subgrid dissipation, transport, pressure strain, and muss flux terms in the Reynolds stress transport equation

$$
\frac{\partial\langle\rho\rangle R_{i j}}{\partial t}+\frac{\partial\langle\rho\rangle \tilde{u}_{k} R_{i j}}{\partial x_{k}}=\langle\rho\rangle\left(P_{i j}-\epsilon_{i j}\right)-\frac{\partial T_{i j k}}{\partial x_{k}}+\Pi_{i j}+M_{i j}
$$

are, respectively 


$$
\begin{aligned}
P_{i j} & =-\left(R_{i k} \frac{\partial\left\langle u_{j}\right\rangle^{f}}{\partial x_{k}}+R_{j k} \frac{\partial\left\langle u_{i}\right\rangle^{f}}{\partial x_{k}}\right) \\
\epsilon_{i j} & =\frac{1}{\langle\rho\rangle}\left\langle\sigma_{j k}^{\prime} \frac{\partial u_{i}^{\prime \prime}}{\partial x_{k}}+\sigma_{i k}^{\prime} \frac{\partial u_{j}^{\prime \prime}}{\partial x_{k}}\right\rangle, \\
\epsilon_{i j}^{\mathrm{sg}} & =\frac{1}{\langle\rho\rangle}\left\langle\tau_{j k}^{\prime} \frac{\partial u_{i}^{\prime \prime}}{\partial x_{k}}+\tau_{i k}^{\prime} \frac{\partial u_{j}^{\prime \prime}}{\partial x_{k}}\right\rangle, \\
T_{i j k} & =\left\langle\rho u_{i}^{\prime \prime} u_{j}^{\prime \prime} u_{k}^{\prime \prime}\right\rangle+\left\langle p^{\prime} u_{i}^{\prime} \delta_{j k}\right\rangle+\left\langle p^{\prime} u_{j}^{\prime} \delta_{i k}\right\rangle-\left(\left\langle\sigma_{j k}^{\prime} u_{i}^{\prime \prime}\right\rangle+\left\langle\sigma_{i k}^{\prime} u_{j}^{\prime \prime}\right\rangle\right), \\
\Pi_{i j} & =\left\langle p^{\prime}\left(\frac{\partial u_{i}^{\prime \prime}}{\partial x_{j}}+\frac{\partial u_{j}^{\prime \prime}}{\partial x_{i}}\right)\right\rangle, \\
M_{i j} & =\left\langle u_{i}^{\prime \prime}\right\rangle\left(\frac{\partial\left\langle\sigma_{j k}+\tau_{j k}\right\rangle}{\partial x_{k}}-\frac{\partial\langle p\rangle}{\partial x_{j}}\right)+\left\langle u_{j}^{\prime \prime}\right\rangle\left(\frac{\partial\left\langle\sigma_{i k}+\tau_{i k}\right\rangle}{\partial x_{k}}-\frac{\partial\langle p\rangle}{\partial x_{i}}\right)
\end{aligned}
$$

\subsection{Growth rates}

Figure 4 shows the momentum thickness, defined as

$$
\delta_{\theta}(t)=\frac{1}{\rho_{0} \Delta u^{2}} \int_{-\infty}^{\infty}\langle\rho\rangle\left(\frac{1}{2} \Delta u-\tilde{u}_{1}\right)\left(\frac{1}{2} \Delta u+\tilde{u}_{1}\right) \mathrm{d} x_{2} .
$$

After a short initial transient, the mixing layer grows almost linearly with growth rates of $\mathrm{d} \delta_{\theta} / \mathrm{d} t / \Delta u=0.0161$, 0.00785, 0.0065, and 0.006 for cases LES03, LES09, LES10, and LES12, respectively. Incompressible temporally evolving mixing layers exhibit growth rates of approximately 0.016 (Rogers et al. [36]). The case LES03 has a convective Mach number small enough for compressibility effects to be considered negligible, its growth rate of 0.0161 is therefore in excellent agreement with the expected growth rate. The shear layer thickness, normalized with its value at the start of the simulation, is plotted along with experiments and DNS data against the convective Mach number in Fig. 5. The strong reduction with increasing $M_{c}$ is captured by the

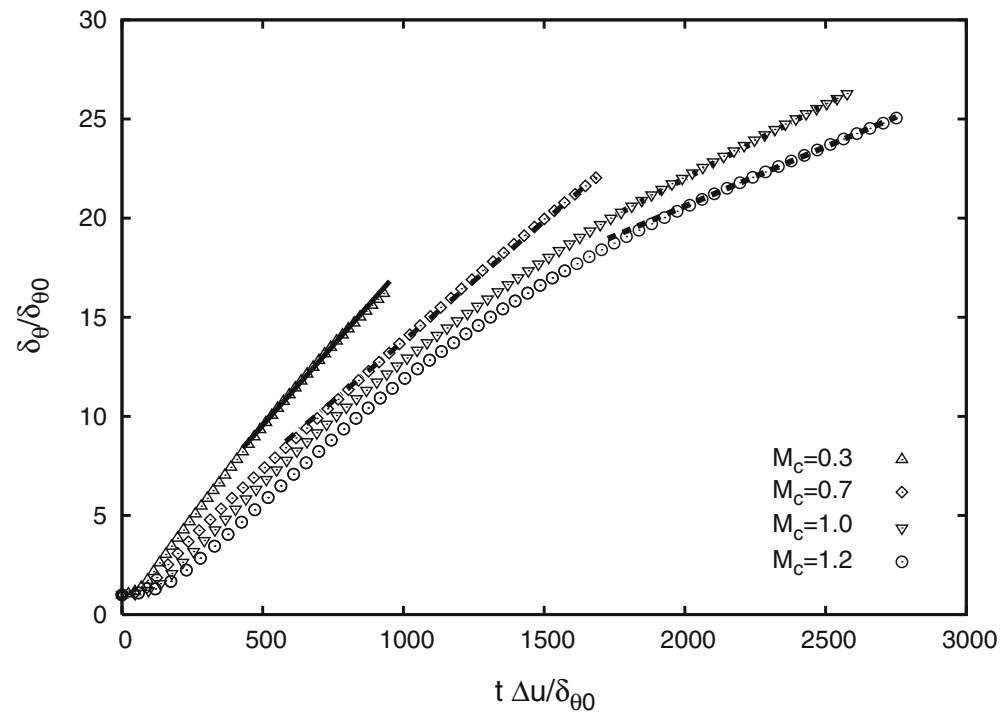

Fig. 4 Time variation of the momentum thickness, normalized with its initial value. The slopes represent the different growth rates in the quasi-self-similar regions, and are 0.0161, 0.0116, 0.00785, 0.0065, and 0.006 for cases LES03, LES07, LES09 (not shown here for clarity), LES10 and LES12, respectively 


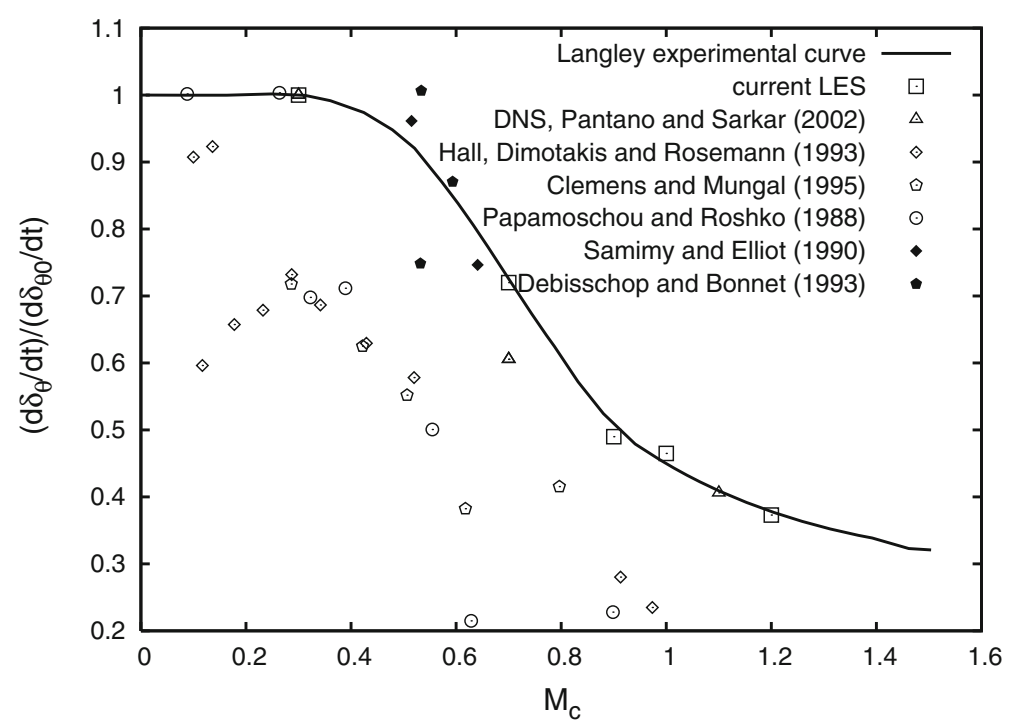

Fig. 5 Shear layer growth rate dependence on $M_{c}$, comparison with experimental and DNS data

present LES. Furthermore, the data is in excellent agreement with the so-called 'Langley Experimental Curve', which is obtained from a compilation of results corresponding to air-air shear layer experiments. Experimental data below the Langley curve are most likely due to density-ratio differences. Another detailed compilation of mixing layer growth rates obtained from various experiments and linear stability analysis can be found in Rossmann et al. [37]. These authors performed experiments at convective Mach numbers ranging from $M_{c}=0.80$ to $M_{c}=2.25$. However, they used different gases and temperatures for the two streams, resulting in a much stronger decrease of the growth rate than observed and predicted by the 'Langley Experimental Curve' and in the numerical simulations reported in this article. As pointed out in the Appendix, a big sample size was necessary to achieve almost self-similarity and get this close agreement between LES and the air-air mixing layer experiments. A smaller domain in the vertical direction results in values which lie further above the Langley curve.

\subsection{Turbulent intensities}

One further check to validate the present LES is comparison of turbulent intensities in the self-similar region (calculated by averaging over profiles plotted in similarity coordinates) seen in Fig. 6, where the turbulent intensity in the streamwise direction $\sqrt{R_{11}} / \Delta u$ is compared to DNS and experimental data. Interestingly, at $M_{c}=0.3$, the LES agrees better with experimental [44] and DNS [36] data in the incompressible shear layer than the DNS results of Pantano et al. [28]. Numerical tests showed the extent of the vertical direction to be a decisive factor, too small a domain led to decreased peak values due to a stronger influence of the boundaries, especially during the late phase of the simulation. It is likely that the DNS would have profited from a somewhat larger vertical domain size. Figures $7 \mathrm{a}-\mathrm{d}$ show the influence of compressibility on the turbulent intensities. As discussed already in the introduction, the peak intensities are reduced with increasing $M_{c}$ as shown in Table 3 .

\subsection{Anisotropy of the Reynolds stresses}

Being part of advanced turbulence closures, the anisotropy tensor,

$$
b_{i j}=\frac{R_{i j}-\frac{2}{3} K \delta_{i j}}{2 K}
$$

is an important characteristic of turbulent flows. As pointed out by [28], previous studies report different results as to whether the diagonal components change as a function of $M_{c}$. Whereas some see only minor changes, other studies indicate that the diagonal components of $b_{i j}$ increase substantially in magnitude, due 


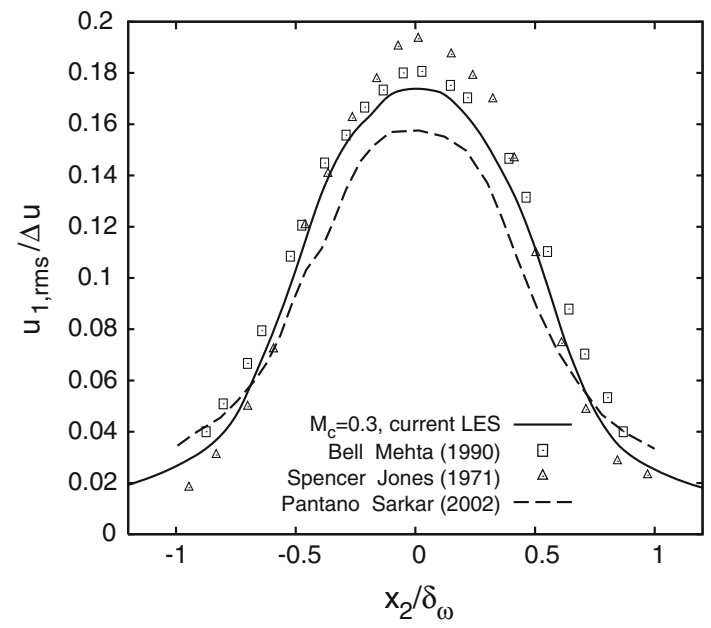

Fig. 6 Comparison of $\sqrt{R_{11}} / \Delta u$ with experiments of [1,44] and DNS simulations [28]
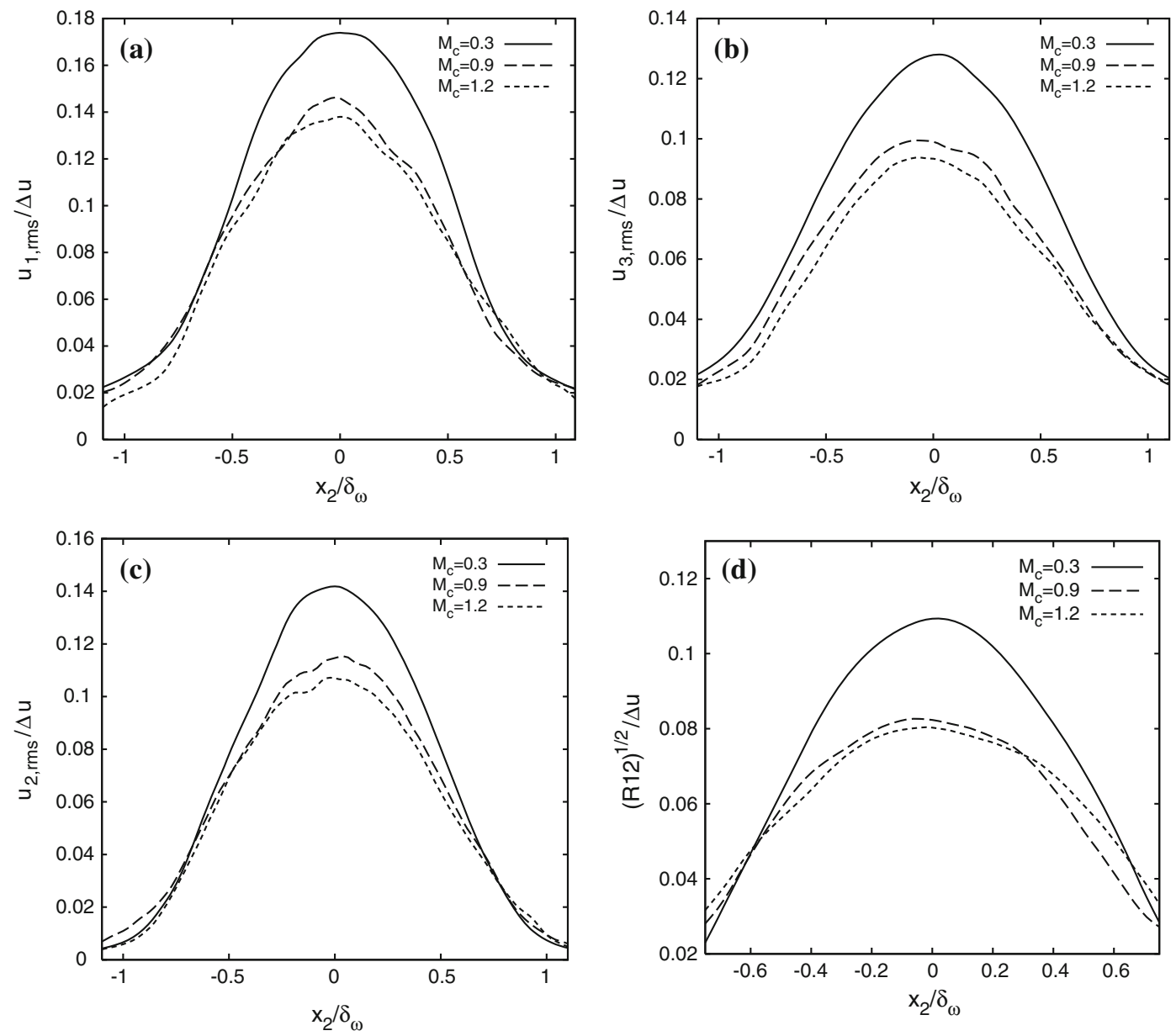

Fig. 7 Profiles of the RMS-velocity and the shear stress for different $M_{c}: \mathbf{a} \sqrt{R_{11}} / \Delta u, \mathbf{b} \sqrt{R_{22}} / \Delta u, \mathbf{c} \sqrt{R_{33}} / \Delta u, \mathbf{d} \sqrt{R_{12}} / \Delta u$

to a stronger decrease in $R_{22}$ and $R_{33}$ than $R_{11}$ with increasing $M_{c}$. Figure 8 shows $b_{11}, b_{22}$ and $b_{12}$, obtained by employing the Reynolds stresses and turbulent kinetic energy, integrated over the shear layer (two vorticity thicknesses), as a function of the non-dimensional time $\tau=t \Delta u / \delta_{\theta 0}$. During an initial transient, a peak whose 
Table 3 Comparison of peak turbulent intensities in experiment, DNS and LES ( $R_{33}$ of [28] not available)

\begin{tabular}{llllllll}
\hline & $M_{c}$ & $\sqrt{R_{11}} / \Delta u$ & $\sqrt{R_{22}} / \Delta u$ & $\sqrt{R_{33}} / \Delta u$ & $\sqrt{R_{12}} / \Delta u$ & $\sqrt{R_{22} / R_{11}}$ & $\sqrt{R_{12} / R_{11}}$ \\
\hline Bell et al. [1] & 0 & 0.18 & 0.14 & 0.146 & 0.100 & 0.777 & 0.555 \\
Present LES & 0.3 & 0.174 & 0.129 & 0.143 & 0.106 & 0.747 & 0.609 \\
Pantano et al. [28] & 0.3 & 0.155 & 0.134 & 0.145 & 0.103 & 0.788 & 0.606 \\
Present LES & 0.9 & 0.146 & 0.10 & 0.115 & 0.084 & 0.685 & 0.575 \\
Pantano et al. [28] & 1.1 & 0.141 & 0.095 & n.a. & 0.083 & 0.674 & 0.588 \\
Present LES & 1.2 & 0.138 & 0.094 & 0.105 & 0.078 & 0.681 & 0.580 \\
\hline
\end{tabular}
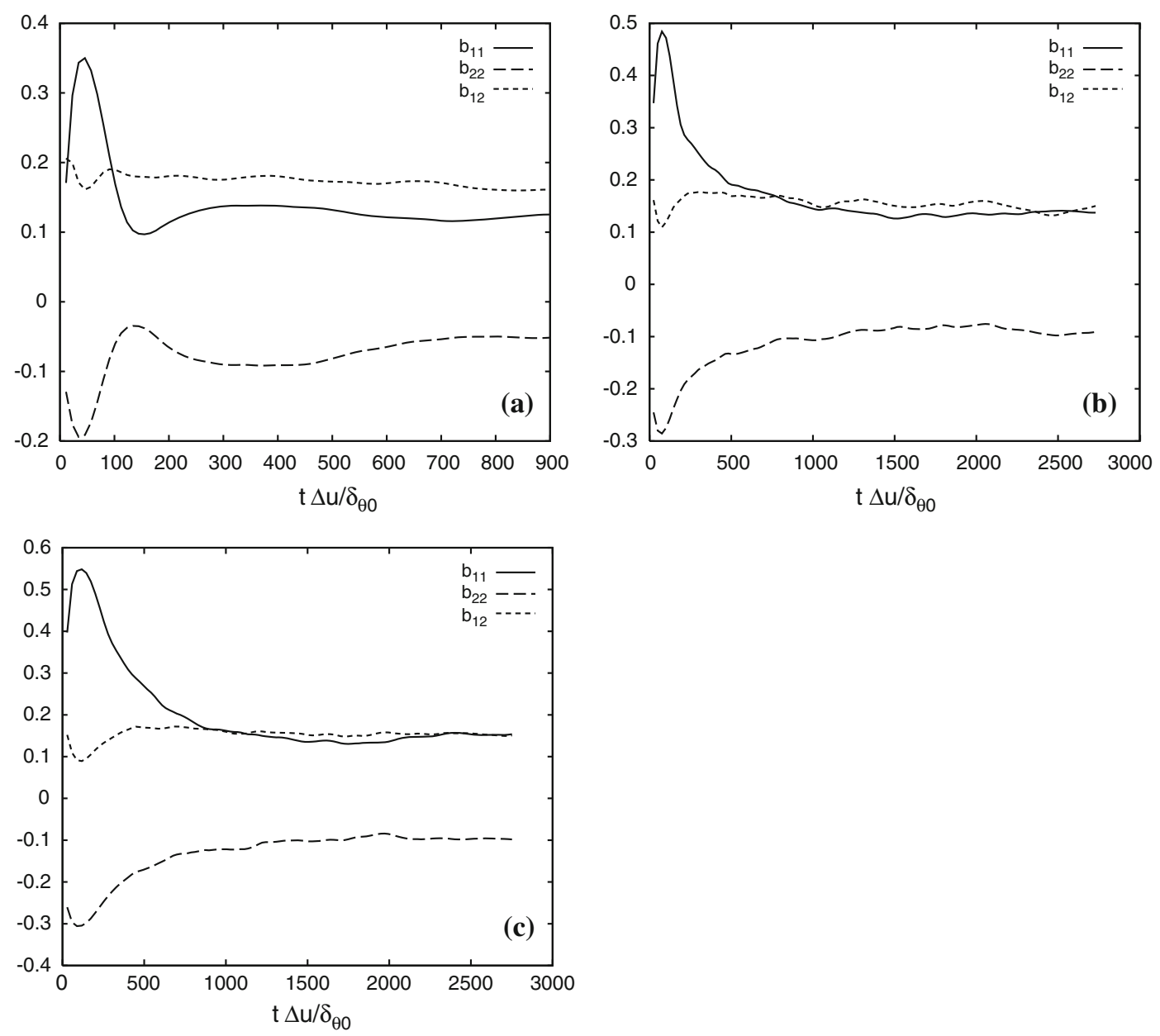

Fig. 8 Evolution of the anisotropy tensor $b_{i j}$ for $\mathbf{a} M_{c}=0.3$, b $M_{c}=0.9$, and $\mathbf{c} M_{c}=1.2$

value strongly depends on the convective Mach number is obtained, before the anisotropies reach an almost asymptotic constant value in the self-similar region. Table 4 shows a comparison of the current LES with DNS results of Pantano et al. [28], for values obtained from the data in the self similar region. A slight increase in magnitude with increasing $M_{c}$ is observed for the diagonal terms $b_{11}$ and $b_{22}$, whereas the DNS data shows an increase only for $b_{22}$, with $b_{11}$ being almost constant. The shear stress anisotropy, $b_{12}$, has negligible change among the different cases, only the DNS data for $M_{c}=0.3$ exhibits a slightly elevated value. The observation of [28], that the anisotropies are strongly affected by $M_{c}$ during their initial evolution, whereas there are only minor changes during their self-similar evolution, holds for the present LES study, too. Furthermore, quantities like $\sqrt{R_{22} / R_{11}}$ and $\sqrt{R_{12} / R_{11}}$, measuring anisotropies and shown in Table 3 for the same time interval, are very similar among the simulations. 
Table 4 Comparison of Reynolds stress anisotropy during the self-similar development in DNS and LES

\begin{tabular}{lllll}
\hline & $M_{c}$ & $b_{11}$ & $b_{22}$ & $b_{12}$ \\
\hline Present LES & 0.3 & 0.13 & -0.05 & 0.16 \\
Pantano et al. [28] & 0.3 & 0.14 & -0.06 & 0.18 \\
Present LES & 0.9 & 0.14 & -0.08 & 0.15 \\
Pantano et al. [28] & 1.1 & 0.14 & -0.10 & 0.16 \\
Present LES & 1.2 & 0.16 & -0.09 & 0.16 \\
\hline
\end{tabular}

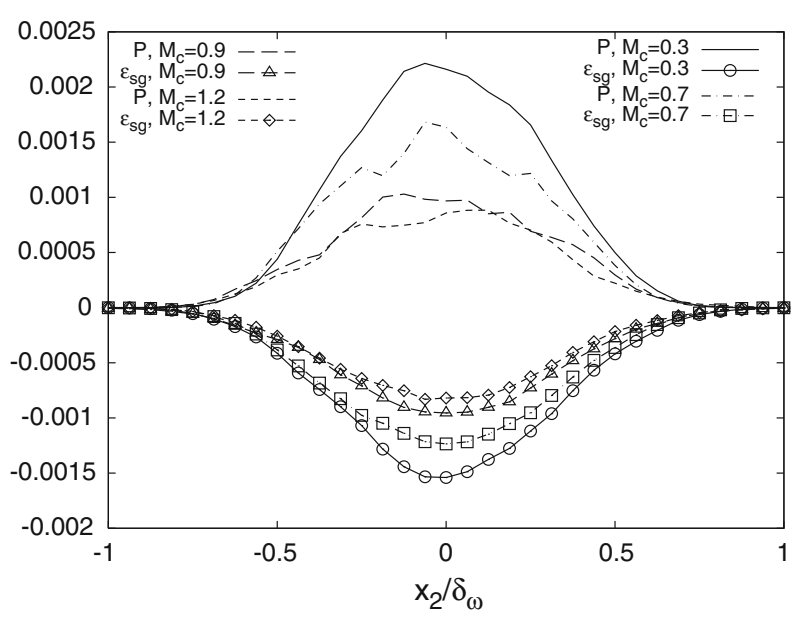

Fig. 9 Production and subgrid dissipation, the dominant terms of the turbulent kinetic energy balance, normalized by $\Delta u^{3} / \delta_{\theta 0}$ for the different LES cases. The resolved dissipation and subgrid production are both negligible

\subsection{Turbulent kinetic energy production and dissipation}

Figure 9 shows the two dominant terms in the turbulent kinetic energy budget, obtained by taking half the trace of the Reynolds stress transport equation. Compared to the subgrid dissipation, the resolved dissipation is negligible. As found in Vreman et al. [50] and later DNS studies, the production term clearly decreases from the case with $M_{c}=0.3$ to $M_{c}=0.9$. The further decrease for case LES12 is small, the same being true for the magnitude of the dissipation. When comparing the turbulent kinetic energy dissipation here with the DNS results in [28], one immediately recognizes the stronger compressibility effect in the present LES relative to the DNS results that showed only a minor influence of compressibility on the dissipation. The extra dissipation is most likely due to filtering, calculated as [28]

$$
\epsilon_{f}=\frac{k-k^{*}}{n \Delta t}
$$

It was estimated to be around 5\% of the actual dissipation in case LES03 and 7\% for LES09 and LES12. Here, $n$ is the number of time steps between filtering and $k^{*}$ denotes the turbulent kinetic energy just after application of the filter.

\section{Resolved-scale vorticity}

Lesieur et al. [19] observed that the coherent vortical structures decrease strongly in size with increasing $M_{c}$, making it necessary to increase the resolution when simulating high- $M_{c}$ flows. The budget for the resolvedscale enstrophy can be of help to understand the generation and change of large-scale vortical structures in a statistical framework. Since a large part of the vorticity is associated with small-scale motion, LES will clearly miss the small scale vorticity contributions to the various terms in the enstrophy budget. Nevertheless, it is interesting to use the LES database to investigate the effect of $M_{c}$ on the resolved vorticity, especially the vorticity production through mean shear and the vortex stretching. We emphasize that all the results in this section apply to the vorticity of the resolved-scale field; DNS is required for inferences about the fine-scale vorticity. da Silva [43], however, demonstrate using DNS and LES of temporal plane jets, that the dynamic Smagorinsky 
model reliably predicts the enstrophy budget, provided that the LES resolutions are at most a factor two to four coarser in each spatial direction, which happens to be the case for the present LES simulations.

The transport equation for the fluctuating resolved-scale enstrophy $\boldsymbol{\omega}^{\prime} \cdot \boldsymbol{\omega}^{\prime} / 2$ is given as $\left.\boldsymbol{\omega}=\left(\omega_{1}, \omega_{2}, \omega_{3}\right)\right)$ :

$$
\begin{aligned}
\frac{\partial}{\partial t} \frac{\boldsymbol{\omega}^{\prime} \cdot \boldsymbol{\omega}^{\prime}}{2}= & \underbrace{\left\langle\omega_{3}\right\rangle\left(\left\langle\omega_{1}^{\prime} \omega_{2}^{\prime}\right\rangle+\left\langle\frac{\partial u_{i}^{\prime \prime}}{\partial x_{3}} \omega_{i}^{\prime \prime}\right\rangle\right)}_{A 1}+\underbrace{\left\langle\omega^{\prime} \cdot \boldsymbol{S}^{\prime} \boldsymbol{\omega}^{\prime}\right\rangle}_{A 2}-\underbrace{\frac{\partial}{\partial x_{2}}\left\langle u_{2}^{\prime} \frac{\left|\boldsymbol{\omega}^{\prime}\right|}{2}\right\rangle}_{A 3} \\
& +\underbrace{\left\langle\left[\nabla \times\left(\frac{1}{\rho} \nabla \cdot \boldsymbol{\sigma}\right)\right]^{\prime} \cdot \boldsymbol{\omega}^{\prime}\right\rangle}_{V 1}+\underbrace{\left\langle\left[\nabla \times\left(\frac{1}{\rho} \nabla \cdot \boldsymbol{\tau}\right)\right]^{\prime} \cdot \omega^{\prime}\right\rangle}_{V 2} \\
& -\underbrace{\left\langle\omega_{3}\right\rangle\left\langle\omega_{3}^{\prime} d^{\prime}\right\rangle}_{D 1}-\underbrace{\left\langle\frac{\left|\boldsymbol{\omega}^{\prime}\right|}{2} d^{\prime}\right\rangle}_{D 2}-\underbrace{\left\langle u_{2}^{\prime} \omega_{3}^{\prime}\right\rangle \frac{\partial\left\langle\omega_{3}\right\rangle}{\partial x_{2}}}_{K}+\underbrace{\left\langle\left[\frac{1}{\rho^{2}} \nabla \rho \times \nabla p\right] \cdot \omega^{\prime}\right\rangle}_{B},
\end{aligned}
$$

denoting production by the mean shear $\left(A 1,\left\langle\omega_{3}\right\rangle=\frac{\partial\left\langle u_{1}\right\rangle}{\partial x_{2}}\right)$, vortex stretching $(A 2)$, transport $(A 3)$, viscous $(V 1)$ and subgrid dissipation ( $V 2)$, vorticity-dilation correlations $(D 1, D 2)$, production by the mean vorticity field $(K)$ and baroclinic production $(B)$, respectively.

Figure 10 shows plots of the different terms for three of the LES cases simulated here. The dominant sources terms are $A 1, A 2$ and the dominant sinks are the subgrid (V2) and filter dissipation $(F D$, defined here as the negative of the sum of the terms in the enstrophy balance). $A 1$ is only marginally affected by compressibility, but the production and subgrid dissipation increase strongly with increasing convective Mach number. Interestingly, term $K$, which describes the exchange of enstrophy between the mean and fluctuating vorticity fields is negligible. The resolved fluctuating enstrophy is therefore mainly produced by stretching and distortion of fluctuating vorticity and, to a lesser extent, by stretching and distortion of the mean vorticity, $\left\langle\omega_{3}\right\rangle$. This effect is intensified with increasing Mach number. The opposite has been observed, for example, in non-rotating and spanwise rotating channel flows [20], where in the near-wall region $A 1$ is the dominant production term, followed by $B 2$. This is of course due to the low Reynolds number near the wall and the high velocity gradients there.

Interestingly, the ratio of $\mathrm{A} 1$ (production by mean shear) to A2 (vortex stretching) seems to decreases dramatically between $M_{c}=0.3$ and 0.9 . This shows that, at higher $M_{c}$, the contribution of the 'linear' mechanism (perhaps related to instabilities) to the amplification of vorticity fluctuations is less important. This is also consistent with a change of large-scale structures between the two cases as seen in Fig. 2 and as stated by [19]. Figure 11a furthermore shows a vorticity correlation length scale, defined as

$$
L_{\omega}=\frac{1}{\left\langle\omega^{\prime 2}\right\rangle} \int d r\left\langle\omega^{\prime}\left(x_{i}+r \delta_{i 2}\right) \omega^{\prime}\left(x_{i}\right)\right\rangle,
$$

which decreases with increasing $M_{c}$ indicating, too, smaller vortical structures. Figure $11 \mathrm{~b}$ shows the vorticity magnitude. Here, a clear increase in the vorticity magnitude with higher $M_{c}$ is seen, contrary to most other turbulent quantities, which decrease. This behaviour goes hand in hand with the increase of the dominant source term A2 in the enstrophy budget. This vortex stretching term can be written as $\boldsymbol{\omega}^{\prime} \cdot\left(\mathbf{S}^{\prime} \boldsymbol{\omega}^{\prime}\right)$. The magnitude of the vector $\mathbf{S}^{\prime} \boldsymbol{\omega}^{\prime}$ and it's orientation with the vorticity vector are shown in Fig. 12 . The magnitude, $\left|S_{i j}^{\prime} \omega_{j}^{\prime}\right|$, turns out to be much larger at higher $M_{c}$, whereas the alignment between the vectors mentioned above changes only to a small degree. Thus, the major increase of $\mathrm{A} 2$ is due to the increased magnitudes of the two vectors. The smaller and increasingly random coherent structures for convective Mach numbers larger than 0.6, as observed in Fig. 2, imply a strong change in the anisotropy of the vorticity field. Figure 13 shows the anisotropy of the vorticity field, defined as $b_{i}^{\omega}=\left\langle\omega_{i}^{\prime 2}\right\rangle /\left\langle\left|\omega^{\prime}\right|\right\rangle$. For high convective Mach number all components obtain values around 0.3 , which indicates equal contributions of all components to the resolved-scale vorticity.

\section{Pressure strain correlation}

As discussed in the introduction, the reduced growth rate of the shear layer has been linked to a reduction in pressure fluctuations and in the pressure-strain correlation. Figure 14 presents the pressure-strain terms $\Pi_{11}$ 

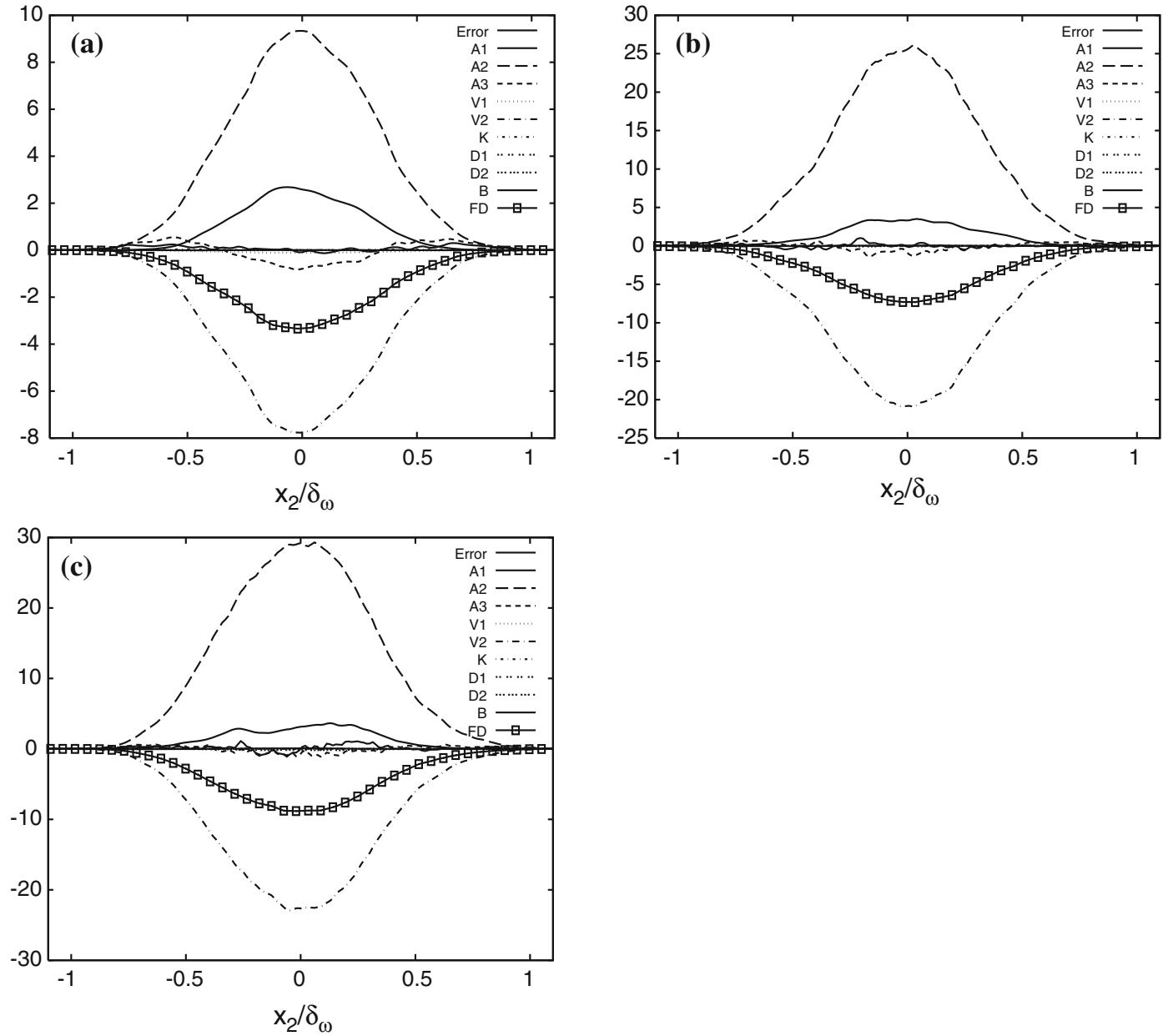

Fig. 10 Profiles of the Enstrophy balance, normalized by $\left(\Delta u / \delta_{\omega}\right)^{3}$ for $\mathbf{a} M_{c}=0.3$, b $M_{c}=0.9$, $\mathbf{c} M_{c}=1.2$
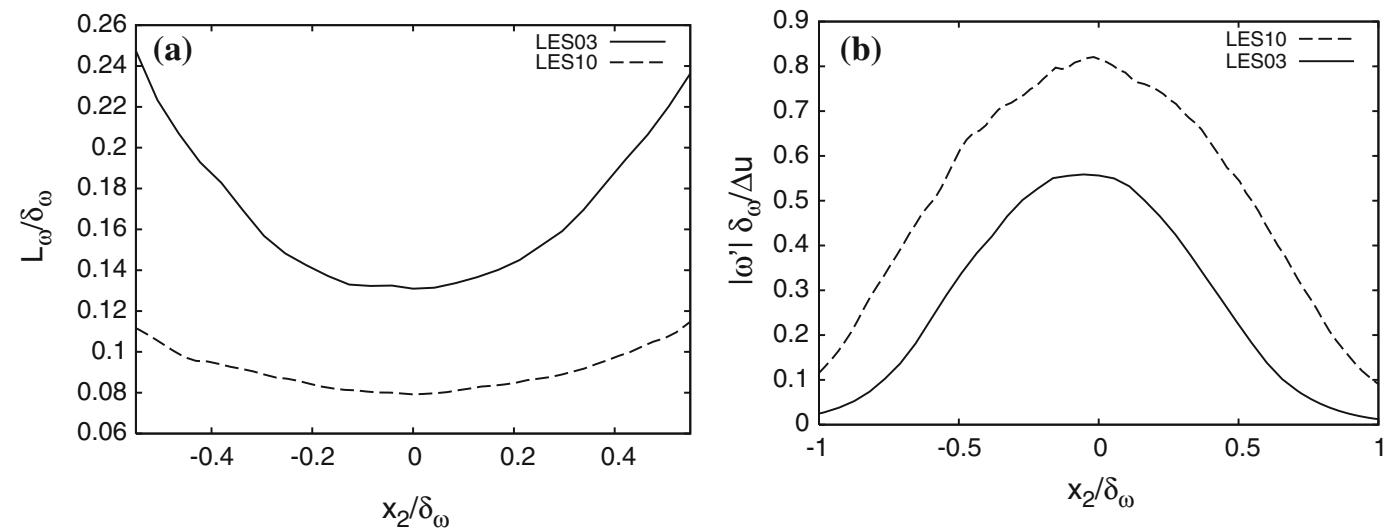

Fig. 11 a Vorticity correlation length-scale $L_{\omega}=\frac{1}{\left\langle\omega^{\prime 2}\right\rangle} \int d r\left\langle\omega^{\prime}\left(x_{i}+r \delta_{i 2}\right) \omega^{\prime}\left(x_{i}\right)\right\rangle$. b Magnitude of the vorticity fluctuations for cases LES03 and LES10

and $\Pi_{22}$, integrated over the shear layer, compared with the DNS data of [28]. The drop of these terms with increasing $M_{c}$ is clearly observed and the agreement with the DNS data is very good. A strong decrease of the pressure fluctuations is also seen. 

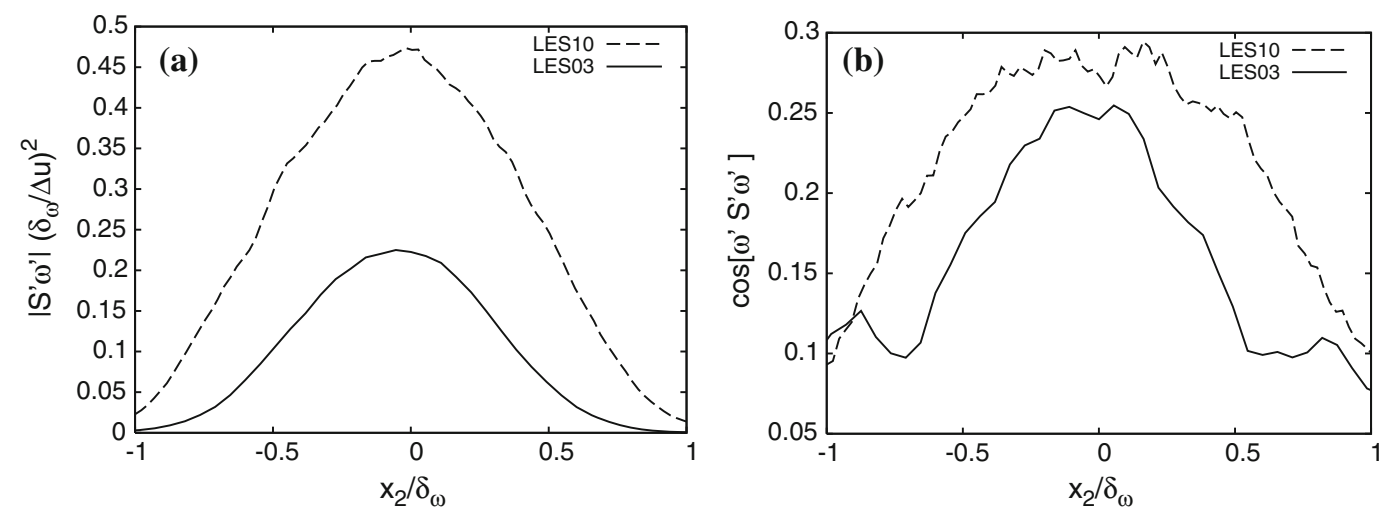

Fig. 12 a $\left|\boldsymbol{S}^{\prime} \boldsymbol{\omega}^{\prime}\right|\left(\delta_{\omega} / \Delta u\right)^{2}$. b Alignment between $\boldsymbol{\omega}^{\prime}$ and $\boldsymbol{S}^{\prime} \boldsymbol{\omega}^{\prime}$ for cases LES03 and LES10
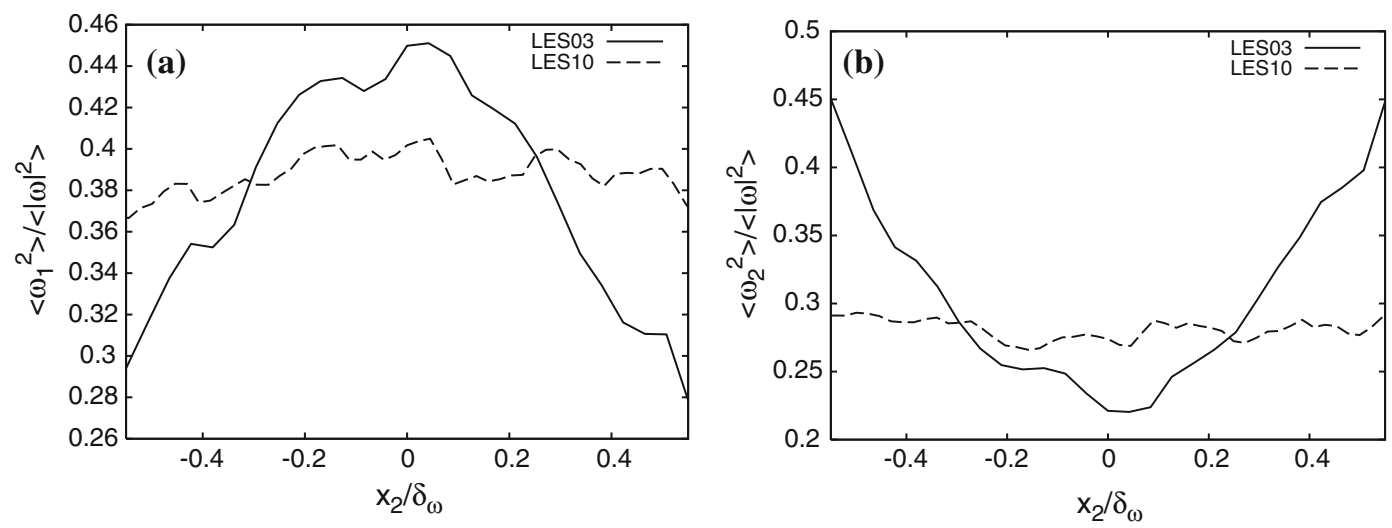

Fig. 13 Vorticity anisotropy $b_{i}^{\omega}=\left\langle\omega_{i}^{2}\right\rangle /\left\langle|\omega|^{2}\right\rangle \mathbf{a} b_{1}^{\omega}, \mathbf{b} b_{2}^{\omega}$ for cases LES03 and LES10

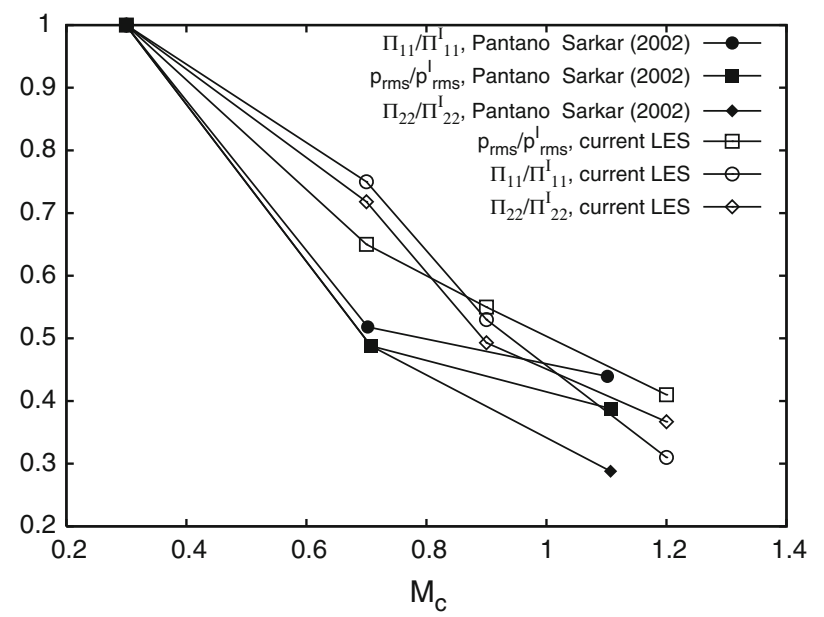

Fig. 14 Dependence of the pressure and pressure-strain terms, integrated over the shear layer, on $M_{C}$

[49] performed a Green's function analysis of the equation governing pressure fluctuations in homogeneous shear flow. The pressure-strain rate spectral function $\Psi_{i j}(\boldsymbol{k})$ is shown to be

$$
\Psi_{i j}(\boldsymbol{k})=2<\rho>\mathcal{G}(\boldsymbol{k})\left(\frac{M_{g}}{M_{t}}\right) k_{l} \lambda_{l m}\left[E_{m i}(\boldsymbol{k}) k_{j}+E_{m j}(\boldsymbol{k}) k_{i}\right]
$$




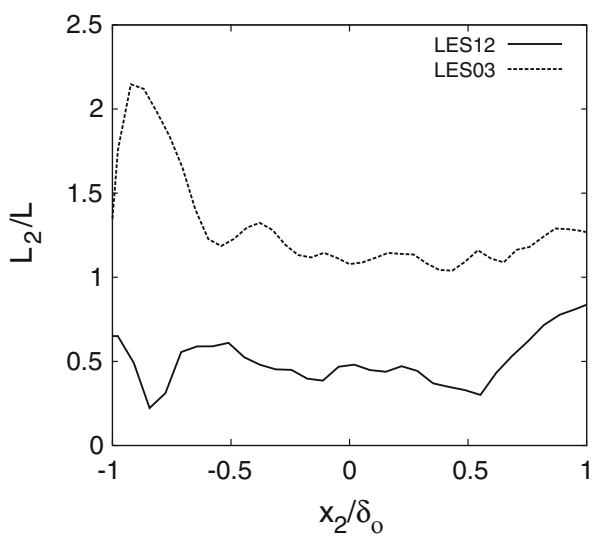

Fig. 15 Comparison of the length scale ratio $L_{2} / L$, as defined in Eq. (21), for cases LES03 and LES12

where $\mathcal{G}(\boldsymbol{k})$ is related to the Green's function and $E_{m i}(\boldsymbol{k})$ is the anisotropic energy spectrum. The authors identify two quantities of importance, the Mach number ratio $\frac{M_{g}}{M_{t}}$ on the one hand and (introduced by a model energy spectrum) a length scale ratio $L_{2} / L$, with

$$
L_{2}=\frac{1}{\left\langle\left(u_{1}^{\prime \prime}\right)^{2}\right\rangle} \int d r\left\langle u_{1}^{\prime \prime}\left(x_{i}+r \delta_{i 2}\right) u_{1}^{\prime \prime}\left(x_{i}\right)\right\rangle, \quad L=K^{3 / 2} / \epsilon
$$

Although the analysis of [49] is not directly applicable to the present inhomogeneous case, the turbulence statistics in the center of the mixing layer have similarities to those in homogeneous shear flow, and it is therefore useful to examine the implications of Eq. 21 using the current database. The Mach number ratio was found to be almost constant between the different LES cases here. Figure 15 on the other hand shows a new result from the LES database: the length scale ratio $L_{2} / L$ clearly reduces from cases LES03 to LES10 which indicates an increased spatial decorrelation with increasing $M_{c}$. The drop is of the same order as the drop seen in the peak of the pressure-strain tensor (Fig. 14). Recall, that the cross-stream correlation length scale of the resolved-scale vorticity, shown earlier in Fig. 11, also showed a substantial reduction with increasing Mach number. A reduction in the vertical extent of the large-scale vortical structures provides a physical link to the reduction of $L_{2} / L$ and, thus, the pressure-strain correlation.

\subsection{Modal decomposition}

Low-wave number (high wavelength) modes in a turbulent flow have larger Mach number based on mean velocity difference or fluctuation velocity difference and are, therefore, expected to be more susceptible to compressibility effects. Low-wave number modes also have an important role in aerodynamic sound. The potential difference between high-and low-wave number modes in the pressure field is assessed in this section. A Fourier decomposition as a function of horizontal wave numbers, $k_{x}, k_{z}$, is performed, modes with wave numbers smaller than a cutoff value are retained in $p^{<}$and the remaining modes are retained in $p^{>}$. Results with two cutoff values, $\left(k_{x}=8, k_{z}=2\right)$ and $\left(k_{x}=12, k_{z}=2\right)$, corresponding to non-dimensional values of $\left(k_{x} \delta_{\theta}=0.96, k_{z} \delta_{\theta}=1.64\right)$ and $\left(k_{x} \delta_{\theta}=1.44, k_{z} \delta_{\theta}=1.64\right)$, respectively, are shown in Fig. 16. Here, $\delta_{\theta}$ is the local value of momentum thickness and the cutoff-wave numbers chosen here separates modes with horizontal wavelength larger than a few momentum thicknesses from those below. The longitudinal integral scale is approximately $2.5 \delta_{\theta}$. Clearly, both low and high- wave numbers contribute to the r.m.s pressure with the contribution of the low-wave number part increasing with increasing value of the cutoff. Furthermore, the low-wave number component, $p^{<}$, has a wider spatial envelope in the cross-stream direction relative to the more compact $p^{>}$field. This is consistent with the low-wave number component being associated with near-field acoustics. Figure 17 shows the pressure-strain component $\Pi_{11}$ for the $M_{c}=1.1$ case, obtained using the unmodified LES data as well as the spectrally decomposed parts $\Pi_{11}^{<}$and $\Pi_{11}^{>}$for the same cutoff-wave numbers employed earlier in Fig. 16 for the r.m.s pressure. Fig. 17a show that the low-wave number modes have negligible contribution to the pressure-strain while increasing the cutoff value in part Fig. 17b still results in a relatively small contribution of the low-wave number modes. Thus, low-wave number modes contribute 

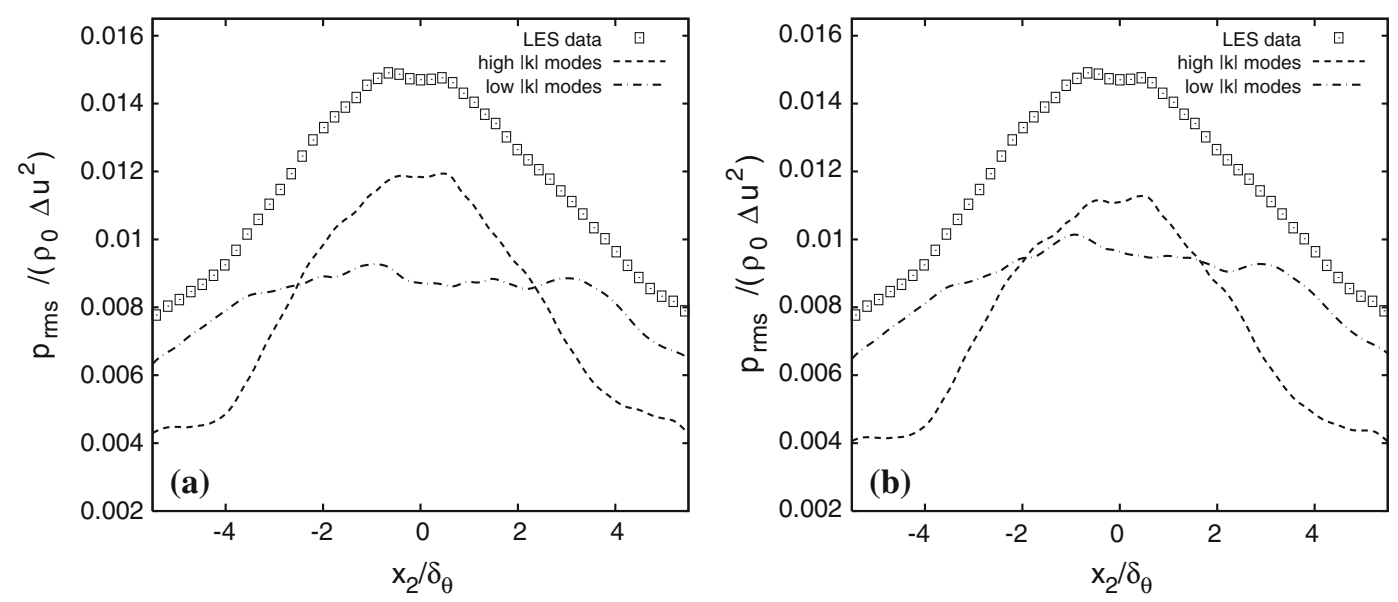

Fig. 16 RMS of pressure fluctuations using all modes (LES data), modes with wavenumbers less than (greater than) a cutoff value of $\mathbf{a}(8,2), \mathbf{b}(12,2)$, respectively, for LES12 with convective Mach number of 1.2
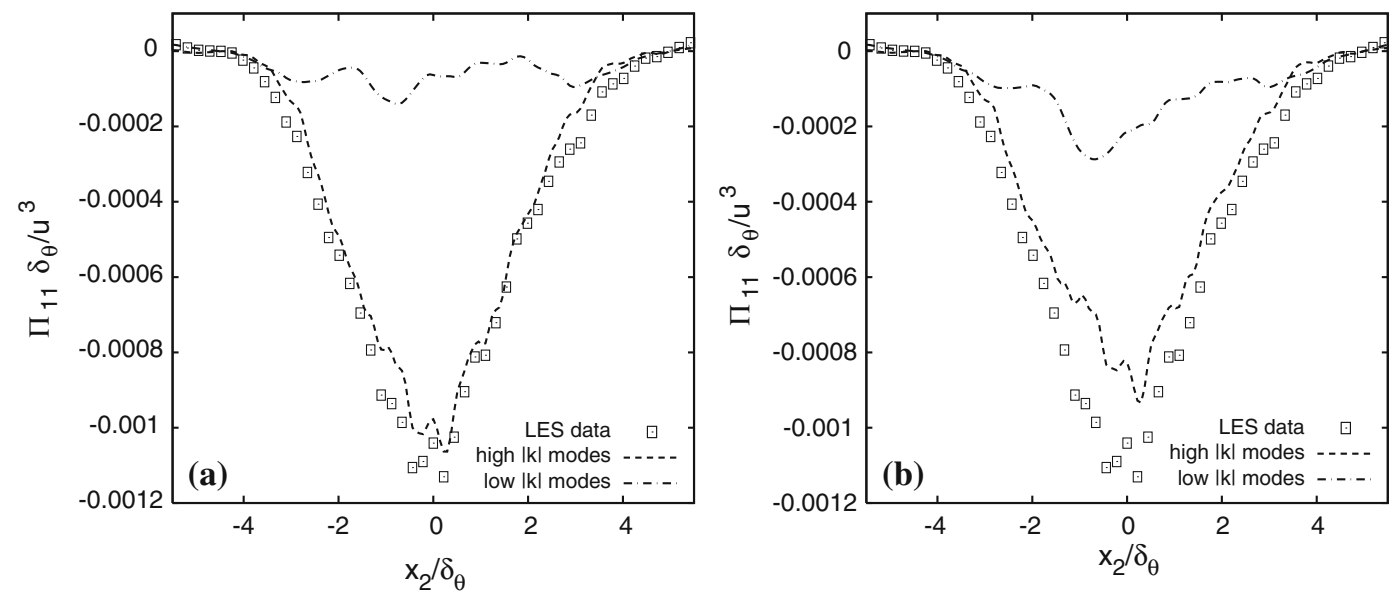

Fig. 17 Pressure-strain terms $\Pi_{11}$ normalized by $\Delta u^{3} / \delta_{\theta}$ using all pressure modes (LES data), modes with wave numbers less than (greater than) a cutoff value of $\mathbf{a}(8,2), \mathbf{b}(12,2)$, respectively, for LES12 with convective Mach number of 1.2

significantly to the r.m.s pressure but do not contribute to the pressure-strain correlation. Examination of the other LES cases show that this conclusion applies to all the Mach numbers simulated here.

A hypothesis for explaining the unimportance of the low-wave number part of the pressure for the pressurestrain term is a low correlation of this part of the pressure with the strain field. If the pressure-strain correlation is rewritten by using the correlation coefficient $\mathcal{C}\left(p^{\prime}, S_{i j}^{\prime}\right)$ as

$$
\Pi_{i j}=\left\langle p^{\prime} S_{i j}^{\prime}\right\rangle=\{p\}_{r m s}\left\{S_{i j}\right\}_{r m s} \mathcal{C}\left(p^{\prime}, S_{i j}^{\prime}\right),
$$

a spectral decomposition of the pressure fluctuations would reveal a reduced correlation for $p^{\prime>}$ with the strain if this hypothesis is true. Figure 18 shows the correlation coefficients $\mathcal{C}\left(p^{\prime}, S_{11}^{\prime}\right)$ and $\mathcal{C}\left(p^{\prime}, S_{33}^{\prime}\right)$ for case LES12. When comparing the correlation coefficient with the ones obtained by using $p^{>}$and $p^{<}$it is evident that $\mathcal{C}\left(p^{\prime>}, S_{i j}^{\prime}\right)$ almost coincides with the complete $\mathcal{C}$, whereas the correlation of the low-wave number component, $p^{\prime<}$, with the strain rate is much lower.

\section{Green's function analysis of the pressure-strain correlation}

Turbulence closures at the level of transport equations for the Reynolds stress tensor require a model for the pressure-strain tensor. There is a long history $[22,33,35,45]$ in the case of incompressible flow wherein the 

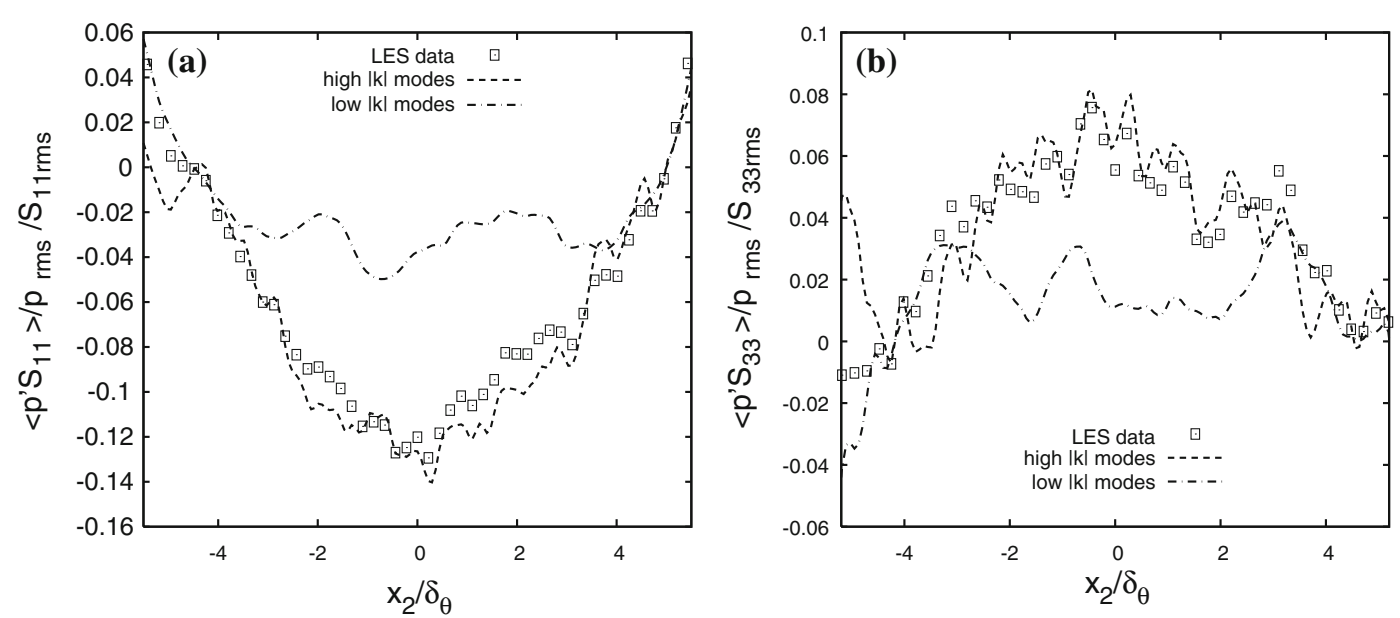

Fig. 18 Correlation coefficient of high- and low-wave number modes with strain rate in LES12. The cutoff value is $(8,2)$ corresponding to $\left(k_{x} \delta_{\theta}=0.96, k_{z} \delta_{\theta}=1.64\right)$. a With $S_{11}$, b with $S_{33}$

pressure-strain tensor is modeled by analysis of a Poisson equation for the pressure. The steps are as follows: obtain a Green's function solution to the Poisson equation for the pressure fluctuations, obtain a formal solution for the pressure-strain correlation by using the Green's function along with the r.h.s of the Poisson equation, and finally, closure by introduction of a model for velocity correlation tensors or, for analysis in spectral space, introduction of a model for the energy spectrum tensor. The objective of this section is not the derivation of a pressure-strain model but is more limited, simply to assess whether the Green's function step can be performed by consideration of a simplified Poisson equation instead of the convected wave equation for pressure. The motivation stems from the LES result of the previous section that "compressible" modes with low wave numbers do not contribute to the pressure-strain correlation.

\subsection{Poisson equation for the pressure}

An equation for the pressure can be obtained by taking the divergence of the momentum equation:

$$
\Delta p=-\left(\rho u_{i} u_{j}\right)_{, i j}+\left(\rho \tau_{i j}\right)_{, i j}+\sigma_{i j, i j}+\frac{\partial^{2} \rho}{\partial t^{2}},
$$

where the over-bar due to filtering has been dropped to ease readability in the following. Using the shorthand $f_{, i}=\frac{\partial f}{\partial x_{i}}$ and inserting the definition of the Reynolds and Favre averages in the Poisson equation above gives

$$
\begin{aligned}
\Delta p^{\prime}= & \left.-2\langle U\rangle_{i, j}^{f}\left(\langle\rho\rangle u_{j}^{\prime \prime}\right)_{, j}-2\langle U\rangle_{i, i}^{f}\left(\langle\rho\rangle u_{j}^{\prime \prime}\right)_{, i}-\left(\langle\rho\rangle u_{i}^{\prime \prime} u_{j}^{\prime \prime}\right)-\left\langle\rho u_{i}^{\prime \prime} u_{j}^{\prime \prime}\right\rangle\right)_{, i j} \\
& -2\langle U\rangle_{i, i j}^{f}\left(\langle\rho\rangle u_{j}^{\prime \prime}\right)-\rho^{\prime}\left(\langle U\rangle_{i, i}^{f}\right)^{2}-\rho^{\prime}\left(\langle U\rangle_{i, j}^{f}\langle U\rangle_{j, i}^{f}\right) \\
& -2\langle U\rangle_{i, j}^{f}\left(\rho^{\prime} u_{j}^{\prime \prime}\right)_{, i}-2\langle U\rangle_{i, i}^{f}\left(\rho^{\prime} u_{j}^{\prime \prime}\right)_{, j}-2\langle U\rangle_{i, i j}^{f}\left(\rho^{\prime} u_{j}^{\prime \prime}\right) \\
& -\left(\rho^{\prime} u_{i}^{\prime \prime} u_{j}^{\prime \prime}\right)_{, i j}+\frac{D^{2}}{D t^{2}} \rho^{\prime}+\sigma_{i j, i j}^{\prime}+\left(\rho \tau_{i j}\right)_{, i j}^{\prime} \\
= & f,
\end{aligned}
$$

with

$$
\begin{aligned}
\frac{D^{2}}{D t^{2}} \rho^{\prime}:= & \left(\frac{\partial^{2}}{\partial t^{2}}+2\langle U\rangle_{j}^{f} \frac{\partial^{2}}{\partial x_{j} \partial t}+\langle U\rangle_{i}^{f}\langle U\rangle_{j}^{f} \frac{\partial^{2}}{\partial x_{i} \partial x_{j}}\right) \rho^{\prime} \\
= & \frac{\partial^{2} \rho^{\prime}}{\partial t^{2}}-2\langle U\rangle_{j}^{f}\left(\rho u_{i}^{\prime \prime}\right)_{i j}-2\langle U\rangle_{j}^{f} \rho^{\prime}\langle U\rangle_{i, i j}^{f} \\
& -2\langle U\rangle_{j}^{f} \rho_{, i}^{\prime}\langle U\rangle_{i, j}^{f}-2\langle U\rangle_{j}^{f} \rho_{, j}^{\prime}\langle U\rangle_{i, i}^{f}-\langle U\rangle_{i}^{f}\langle U\rangle_{j}^{f} \rho_{, i j}^{\prime} .
\end{aligned}
$$




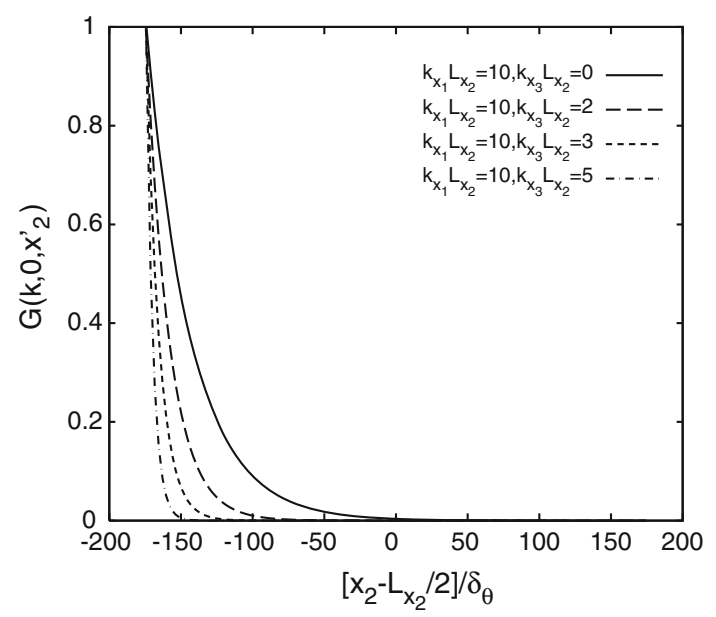

Fig. 19 Green's function for a location at the bottom of the computational domain, normalized to a maximal magnitude of 1

The r.h.s of Eq. 24 involves terms dependent on the mean density, fluctuations in density, viscosity and the subgrid stress. If an isentropic relation $p^{\prime}=\langle c\rangle^{2} \rho^{\prime}$ is used to relate the pressure to the density fluctuations following a given fluid particle, a convective wave equation for the pressure fluctuation can be obtained. An alternate viewpoint is to treat the second derivative of the density fluctuations as an additional source term on the r.h.s of a Poisson equation. Although the interpretation of a wave equation for the pressure is lost, the advantage is an explicit analytical solution for the pressure as show below. The pressure field is given by the following formal solution of the Poisson equation after introduction of a Green's function:

$$
p^{\prime}\left(x_{1}, x_{2}, x_{3}\right)=\int_{0}^{L_{x_{2}}} G * f\left(x_{1}, x_{2}, x_{3} ; x_{2}^{\prime}\right) \mathrm{d} x_{2}^{\prime}
$$

where the convolution $G * f$ is the inverse Fourier transform of $\hat{G} \hat{f}$ i.e,

$$
G * f\left(x_{1}, x_{2}, x_{3} ; x_{2}^{\prime}\right)=\frac{1}{(2 \pi)^{2}} \int_{-\infty}^{\infty} \mathrm{d} k_{1} \int_{-\infty}^{\infty} \mathrm{d} k_{3} \exp (i \mathbf{k} \cdot \mathbf{x}) \hat{G}\left(k, x_{2} ; x_{2}^{\prime}\right) \hat{f}\left(k_{1}, k_{3} ; x_{2}^{\prime}\right),
$$

and a closed-form solution for $\hat{G}$ can be written, for example, as in Kim [16]. The Green's function, $G\left(k_{x_{1}}, k_{x_{3}}, x_{2}, x_{2}^{\prime}\right)$, peaks at $x_{2}=x_{2}^{\prime}$ and decays with increasing distance, $r_{2}=\left|x_{2}-x_{2}^{\prime}\right|$, from the source region. The rate of decay decreases with decreasing magnitude of horizontal wave number as shown in Fig. 19, indicating that the cross-stream extent of the domain of influence of the Green's function increases with increasing horizontal length scale of the chosen spectral mode.

After introduction of the Green's function, the pressure-strain correlation can be written as

$$
\Pi_{i j}\left(x_{2}\right)=\int_{0}^{L_{x_{2}}}\left\langle G * f^{\prime}\left(x_{1}, x_{2}, x_{3} ; x_{2}^{\prime}\right) s_{i j}^{\prime}\right\rangle d x_{2}^{\prime}
$$

The quantity, $\left\langle G * f\left(x_{1}, x_{2}, x_{3} ; x_{2}^{\prime}\right) s_{i j}^{\prime}\right\rangle$, which is a function of the observer cross-stream position, $x_{2}$, and the source cross-stream position, $x_{2}^{\prime}$, is numerically obtained by averaging the instantaneous values of $G *$ $f\left(x_{1}, x_{2}, x_{3} ; x_{2}^{\prime}\right) s_{i j}^{\prime}$ over $x_{1}, x_{3}$ planes as well as ensembles at different times.

\subsection{Pressure-strain correlation using the Green's function}

In the following analysis, we will solve the Poisson equation (24) using the incompressible Green's function and treating the second derivatives of the density fluctuation as an additional source term. The LES database 

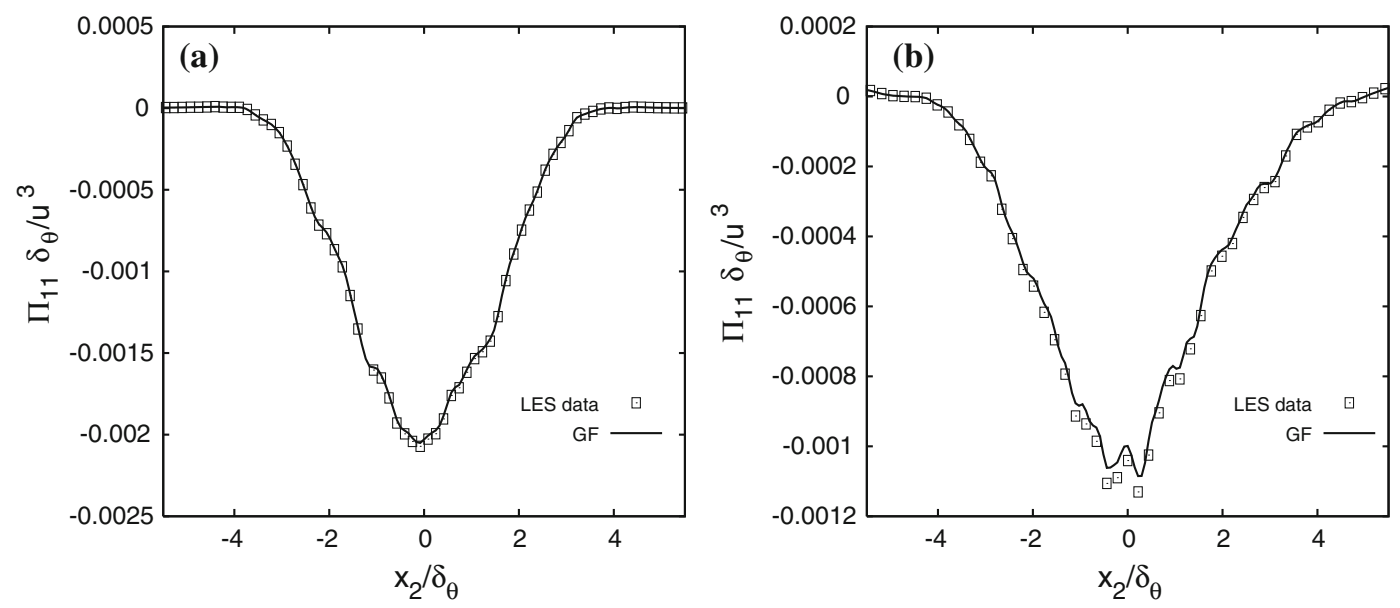

Fig. 20 Pressure strain terms $\Pi_{11}$ normalized by $\Delta u^{3} / \delta_{\theta}$. Comparison of Green's function solution and the LES data. a $M_{c}=0.7$, b $M_{c}=1.2$

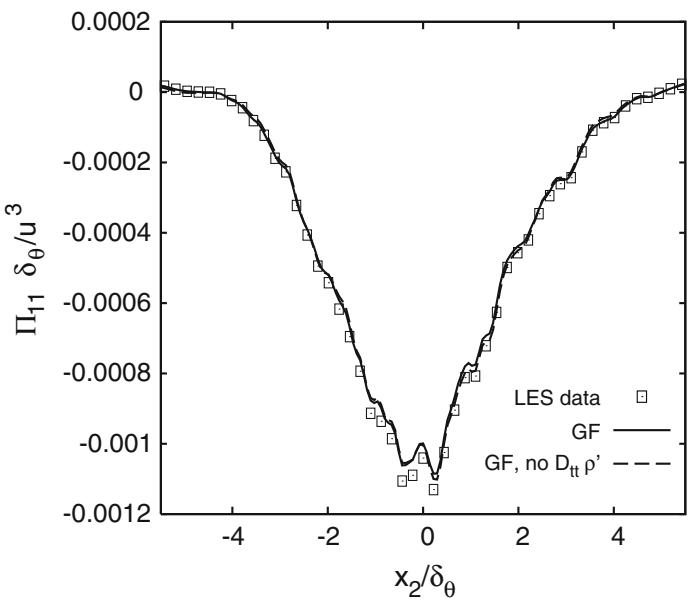

Fig. 21 Influence of the density fluctuation term, $D^{2} \rho^{\prime} / t^{2}$, on the Green's function solution for the pressure-strain correlation. Case with $M_{c}=1.2$ is shown

enables us to calculate the source term and thus evaluate the merit of the Poisson equation assumption with respect to the pressure-strain correlation.

Figure 20 shows the 11-component of the pressure-strain correlation for $M_{c}=0.7$ and 1.2. The Green's function solution, Eq.28, is in very good agreement with the pressure-strain correlation extracted directly from the LES data.(The same is true for the other components of $\Pi_{i j}$, not shown here). It is possible to make a further simplification by dropping the term, $D^{2} \rho^{\prime} / D t^{2}$, from the r.h.s. of the Poisson equation for the pressure. As shown by Fig. 21, dropping this term has no impact on the pressure-strain correlation. Thus, $D^{2} \rho^{\prime} / D t^{2}$, the term from which the wave equation for pressure follows, has little influence on the pressure-strain correlation. It has to be emphasized that the simplification of a Poisson equation for the pressure applies to the special case of pressure-strain correlation; the contribution of the low wave number "compressible" modes to the r.m.s pressure is significant as was shown earlier in Fig. 16.

\section{Conclusions}

Large eddy simulations of temporally developing mixing layers were conducted with convective Mach numbers ranging from 0.3 to 1.2 and Reynolds numbers based on the vorticity thickness as high as 20,000 during the self-similar stage. A large computational domain was chosen to ensure a sample size sufficiently large to achieve converged statistics. The cross-stream extent of the domain was found to be especially crucial in 


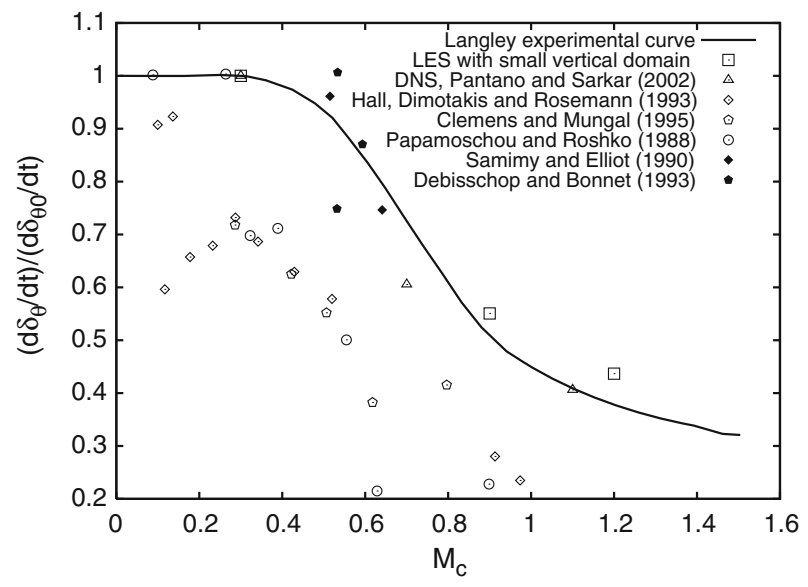

Fig. 22 Shear layer growth rate comparison with experimental and DNS data
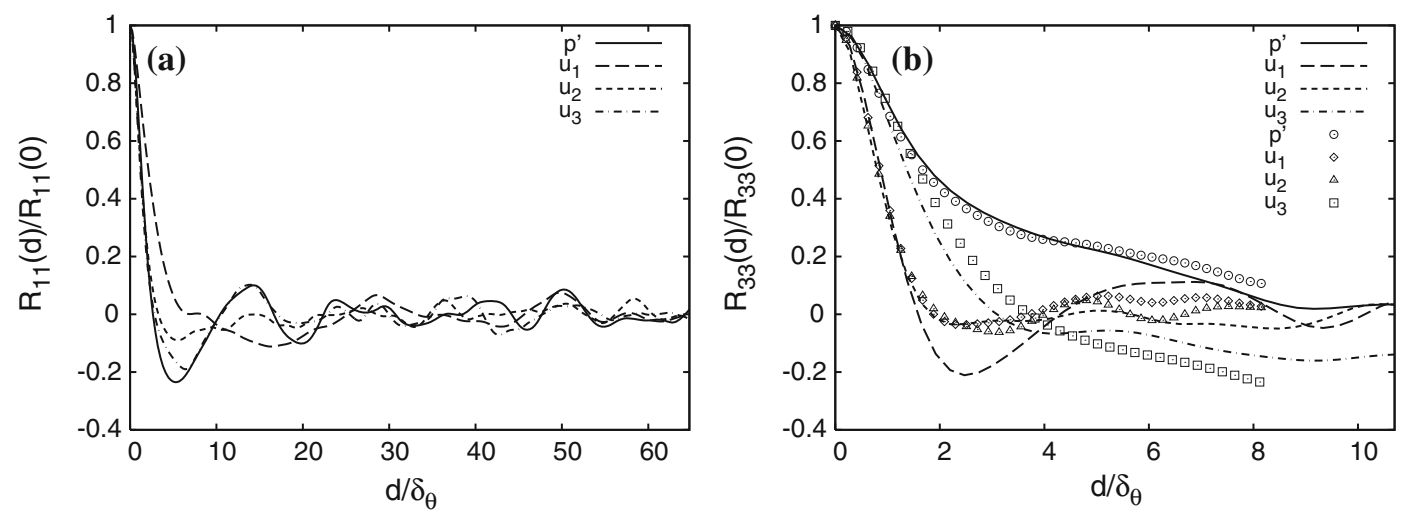

Fig. 23 Two-point correlations as a function of the separation $d$, at the mixing layer center: a streamwise and $\mathbf{b}$ spanwise direction. In part b results from case LES03 with a spanwise domain size of $L_{3}=118 \delta_{\theta, 0}$, shown in symbols, are compared with those of the present simulation with larger spanwise domain size, $L_{3}=172 \delta_{\theta, 0}$
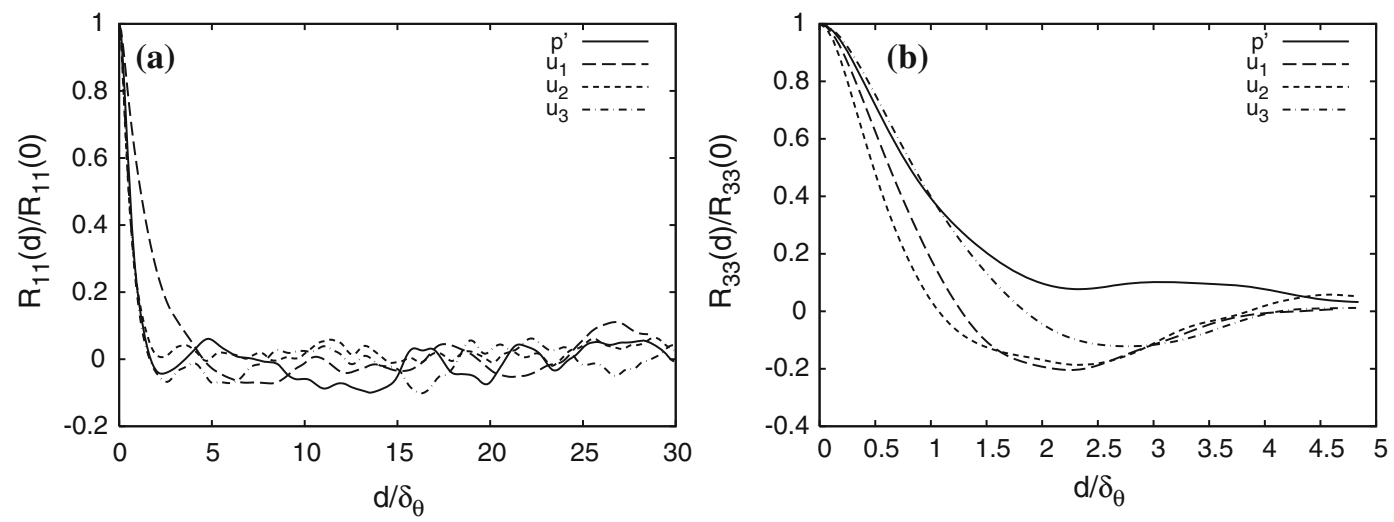

Fig. 24 Two-point correlations in the streamwise (a) and spanwise (b) directions as a function of the separation $d$, at the mixing layer center, for case LES12

order to minimize the effect of cross-stream boundaries on the growth rate and other turbulent quantities. All simulations were initialized using the same broadband disturbances and showed, consistent with previous work, transition scenarios that change with Mach number. The suppression of the Kelvin-Helmholtz instability for convective Mach numbers larger than 0.6 resulted in smaller and streamwise elongated, less 
coherent pressure tubes. As a consequence, a higher resolution in high Mach number simulations is required for accuracy.

The present simulations clearly show that, when properly conducted, LES can accurately represent compressibility effects in a mixing layer. The study is not a DNS because the grid size is two orders of magnitude larger than the Kolmogorov scale and the resolved-scale molecular dissipation is negligible compared to the dissipation provided by the subgrid model. Nevertheless, the LES cell size is sufficiently small for good resolution of the structures that carry turbulent kinetic energy and Reynolds shear stress. The decrease of the momentum thickness with increasing $M_{c}$ was captured, and the resulting growth rates obtained during the quasi-self-similar phase showed excellent agreement with the Langley experimental curve, a compilation of air-in-air measurements of mixing layers. It is worth noting that there are data sets with growth rates less than the Langley curve. Some of the differences might be due to a difference in composition and density between the free streams. Previous DNS of a shear layer between streams with different density has shown that unequal densities can reduce the growth rate but further LES and laboratory studies that allow access to a wide composition/density space at fixed $M_{c}$ are desirable. Comparison of the present LES results with DNS data of [28] and experiments of [1] in the canonical air-air shear layer revealed excellent agreement of the Reynolds stresses and their anisotropies. Furthermore, the decay of the pressure fluctuations and the pressure strain terms with increasing $M_{c}$ agrees well with the DNS database. Unlike conventional Reynolds-averaged closures, no explicit compressibility modifications were required to the LES model in order to capture the influence of $M_{c}$ on the flow.

The vorticity of the resolved-scale field was analyzed in a statistical framework to investigate large-scale vortical structures that are thought to be related to entrainment, growth rate and Reynolds stresses. Comparison of the production terms in the balance of resolved-scale enstrophy led to an interesting result. With increasing Mach number, the ratio of the production by mean shear to the production by vortex stretching decreased strongly. This indicates that the amplification of vorticity fluctuations by linear effects (related to perhaps large-scale instabilities) becomes less important with increasing Mach number. Visualizations clearly showed that, consistent with previous linear analysis, there is a different route to turbulence at high $M_{c}$ that results in smaller, less coherent vortices. The resolved-scale vorticity was found to grow in magnitude with increasing Mach number contrary to the other turbulent quantities which decrease. Insofar as the nonlinear vortex stretching term $\omega_{i}^{\prime} S_{i j}^{\prime} \omega_{j}^{\prime}$, the magnitude of $\left|S_{i j}^{\prime} \omega_{j}^{\prime}\right|$ increased with increasing $M_{c}$ while there was little change in the alignment between the fluctuating vorticity vector and the action of the strain on the vorticity $S_{i j}^{\prime} \omega_{j}^{\prime}$. In addition, at high Mach numbers, the anisotropy of the resolved-scale vorticity field, measured by the relative contribution of each component to the r.m.s fluctuation, was found to decrease, consistent with the lack of organized Kelvin-Helmholtz rollers.

The pressure-strain correlation has received attention in previous simulations and theoretical analysis of compressible turbulence. The vertical correlation length scale of the two-point velocity correlation, when normalized by an isotropic estimate, is observed to decrease in the present LES. Such a decrease in length scale ratio, when input into the analytical formula of [49], would lead to a decrease in the pressure-strain correlation. The vertical length scale of the two-point correlation of the resolved-scale vorticity is found to decrease with increasing $M_{c}$. These results from the LES, when combined with previous laboratory results showing increased three-dimensionalization and smaller vortical structures, show that reduced spatial correlation of turbulence at high $M_{c}$ is an important contributor to the observed compressibility effect of reduced pressure-strain correlation.

A modal decomposition of the pressure fluctuations was employed and it was found that low-wave number modes contribute to the r.m.s pressure but not the pressure-strain correlation. The reason is the low correlation coefficient between the 'compressible' low-wave number modes and the fluctuating strain rate. A model of the pressure-strain correlation is required in turbulence closures at the level of Reynolds stress transport equations. The use of a Green's function for the Poisson equation for pressure is a customary starting point for development of pressure-strain models in incompressible flow. It is demonstrated using the LES database that, although the flow studied here is compressible, the Poisson equation simplification, neglecting the term $D^{2} \rho^{\prime} / D t^{2}$, is valid insofar as the pressure-strain correlation is concerned. The reason is that the term $D^{2} \rho^{\prime} / D t^{2}$, the one that leads to a wave equation for pressure after assuming isentropy, contributes solely to the low wave number modes of pressure which, as discussed earlier, have negligible contribution to the pressure-strain correlation. Use of a simplified Green's function as in this article, instead of the complicated Green's function of [49] for a convected wave equation, would lead to simpler and more tractable functional forms for pressure-strain models. 


\section{Appendix A: A effect of domain-size}

Different resolutions and domain sizes were tested, based on the original simulation domain of [28]. The large number of points used here was necessary to obtain smooth and converged statistics. Using the domain sizes of [28] with a grid resolution as given in Table 2 did not show self-similar growth for the cases with $M_{c}>0.7$. Quasi-self-similar behavior was obtained on the other hand when increasing the sample size. The statistics converged after increasing the vertical extent of the box successively, leading to the growth rates and values given in this article. Figure 22 shows growth rates obtained for a small vertical domain size, $L_{2} / \delta_{\theta_{0}}=200$. The influence of the boundaries produces larger growth rates than the ones compiled in the Langley experimental curve. The large-domain LES results analyzed in the body of this article led to very good agreement with the Langley curve as shown earlier in Fig. 5.

\section{Appendix B: Two-point correlations}

Figures 23 and 24 show the two-point correlations, $R_{i i}^{u_{i}}=\left\langle u_{i}\left(\boldsymbol{x}+\boldsymbol{e}_{i} d\right) u_{i}(\boldsymbol{x})\right\rangle$, at the time corresponding to the start of the self-similar behavior, plotted as a function of the separation $d$ normalized with the momentum thickness at that time. The streamwise correlations show excellent decrease and the spanwise correlations show sufficient decrease with increasing separation, thus validating the chosen box size. For case LES03, we furthermore tested two different domain sizes, $L_{3}=118 \delta_{\theta, 0}$ and $172 \delta_{\theta, 0}$ in the span-wise direction. There was little effect of spanwise domain size on the evolution of shear layer thickness and turbulence intensities.

Acknowledgements The first author was supported by a Feodor-Lynen Research Fellowship of the Humboldt-Foundation in Germany.

Open Access This article is distributed under the terms of the Creative Commons Attribution Noncommercial License which permits any noncommercial use, distribution, and reproduction in any medium, provided the original author(s) and source are credited.

\section{References}

1. Bell, J., Mehta, R.: Developement of a two-stream mixing layer from tripped and untripped boundary layers. AIAA J. 28, 2034-2042 (1990)

2. Berland, J., Bogey, C., Bailly, C.: Low-dissipation and lowdispersion fourth-order Runge-Kutta algorithm. Comput. Fluids, 1459-1463 (2006)

3. Bogdanoff, D.: Compressibility effects in turbulent shear layers. AIAA J. 21, 926-927 (1983)

4. Bogey, C., Bailly, C.: Large eddy simulation of round free jets using explicit filtering with/without dynamic Smagorinsky model. Int. J. Heat Fluid Flow 27, 603-610 (2006)

5. Bradshaw, P.: Compressible turbulent shear layers. Ann. Rev. Fluid Mech. 21, 926-927 (1977)

6. Cambon, C., Coleman, G. N., Mansour, N. N.: RDT and DNS of compressible homogeneous turbulence at finite Mach number. J. Fluid Mech. 257, 641-665 (1993)

7. Clemens, N., Mungal, M.: Two- and three-dimensional effects in the supersonic mixing layer. AIAA J. 30, 973-981 (1992)

8. Clemens, N., Mungal, M.: Large-scale structure and entrainment in the supersonic mixing layer. J. Fluid Mech. 284, 171-216 (1995)

9. Elliott, G., Samimy, M.: Compressiblity effects in free shear layers. Phys. Fluids A(2), 1231-1240 (1990)

10. Erlebacher, G., Hussaini, M., Kreiss, H., Sarkar, S.: The analysis and simulation of compressible turbulence. Theor. Comput. Fluid Dyn. 2, 73-95 (1990)

11. Favre, A.: Turbulence: space-time statistical properties and behaviour in supersonic flows. Phys. Fluids 26, 2851-2863 (1983)

12. Foysi, H., Sarkar, S., Friedrich, R.: Compressibility effects and turbulence scalings in supersonic channel flow. J. Fluid Mech. 509, 207-216 (2004)

13. Freund, J., Lele, S., Moin, P.: Compressibility effects in a turbulent annular mixing layer. Part 1. Turbulence and growth rate. J. Fluid Mech. 421, 229-267 (2000)

14. Freund, J., Lele, S., Moin, P.: Compressibility effects in a turbulent annular mixing layer. Part 2. Part mixing of a passive scalar. J. Fluid Mech. 421, 269-292 (2000)

15. Johansson, S.: High order finite difference operators with the summation by parts property based on DRP schemes. Technical Report 2004-036 (2004)

16. Kim, J.: On the structure of pressure fluctuations in simulated turbulent channel flow. J. Fluid Mech. 205, 421-451 (1989)

17. Kline, S.J., Cantwell, B. J., Lilley, G. M.: Procceding 1980-81-AFOSR-HTTM-Stanford Conference on Complex Turbulent Flows, Vol. 1. Stanford University Press, Stanford (1982)

18. Lele, S. K.: Compressibility effects on turbulence. Ann. Rev. Fluid Mech. 26, 211-254 (1994)

19. Lesieur, M., Metais, O., Comte, P.: Large-Eddy Simulations of Turbulence. Cambridge University Press, Cambridge (2005)

20. Liu, N. S., Lu, X. Y.: A numerical investigation of turbulent flows in a spanwise rotating channel. Comput. Fluids 36, 282-298 (2007) 
21. Lui, C.: Numerical investigation of shock-associated noise. PH.D-Thesis, Stanford University (2003)

22. Lumley, J. L.: Computational modeling of turbulent flows. Adv. Appl. Mech. 18, 123-176 (1978)

23. Mahle, I., Friedrich, R., Foysi, H., Sarkar, S.: Effects of heat release on the turbulence structure in temporally evolving compressible mixing layers. Proceedings of the Turbulent Shear Flow Phenomena 5, Munich (2007)

24. Mathew, J., Lechner, R., Foysi, H., Sesterhenn, J., Friedrich, R.: An explicit filtering method for large eddy simulation of compressible flows. Phys. Fluids 15(8), 2279-2289 (2003)

25. Mattsson, K., Nordström, J.: Summation by parts operators for finite difference approximations of second derivatives. J. Comput. Phys. 199, 503-540 (2004)

26. Moin, P., Squires, K., Cabot, W., Lee, S.: A dynamic subgrid-scale model for compressible turbulence and scalar transport. Phys. Fluids A3(11), 2746-2757 (1991)

27. Normand, X.: Transition a la turbulence dans les ecoulements cisailles libres et parieteaux. PhD Thesis, Grenoble (1990)

28. Pantano, C., Sarkar, S.: A study of compressibility effects in the high-speed, turbulent shear layer using direct simulation. J. Fluid Mech. 451, 329-371 (2002)

29. Papamoschou, D.: Structure of the compressible turbulent shear layer. AIAA Paper 89-0126 (1989)

30. Papamoschou, D.: Zones of influence in the compressible shear layer. Fluid Dyn. Res. 11, 217-228 (1993)

31. Papamoschou, D., Lele, S.: Vortex-induced disturbance field in a compressible shear layer. Phys. Fluids A 5, 1412-1419 (1993)

32. Papamoschou, D., Roshko, A.: The compressible turbulent mixing layer: an experimental study. J. Fluid Mech. 197, 453-477 (1988)

33. Pope, S. B.: Turbulent flows. Cambridge University Press, Cambridge (2000)

34. Ragab, A., Wu, J.: Linear instabilities in two dimensional compressible mixing layers. Phys. Fluids A(6), 957-966 (1989)

35. Reynolds, W. C.: Computation of turbulent flows. Annu. Rev. Fluid Mech. 8, 183-208 (1976)

36. Rogers, M., Moser, R.: Direct simulation of a self-similar turbulent mixing layer. Phys. Fluids 6, 903-923 (1994)

37. Rossmann, T., Mungal, M., Hanson, R.: Evolution and growth of large-scale structures in high compressibility mixing layers. J. Turbul. 3, 009 (2002)

38. Sagaut, P.: Large Eddy Simulation for Incompressible Flows: An Introduction. 2nd edn. Springer Verlag, New York (2002)

39. Sandham, N. D., Reynolds, W.: Compressible mixing layer: linear theory and direct simulation. AIAA J. 28, 618-624 (1990)

40. Sarkar, S.: The stabilizing effect of compressibility in turbulent shear flow. J. Fluid Mech. 282, 163-186 (1995)

41. Sarkar, S.: On density and pressure fluctuations in uniformly sheared compressible flow. In: L. Fulachier, J. L. Lumley, F. Anselmet (eds.) Proceedings, IUTAM Symposium on Variable Density Low-Speed Flows, Marseille (1996)

42. Shao, L., Sarkar, S., Pantano, C.: On the relationship between the mean flow and subgrid stresses in les of turbulent shear flows. Phys. Fluids 11, 1229-1248 (1999)

43. da Silva, C.B., Pereira, J.C.F.: The effect of subgrid-scale models on the vortices computed from large-eddy simulations. Phys Fluids 16(12), 4506-4534 (2004). doi:10.1063/1.1810524. http://link.aip.org/link/?PHF/16/4506/1

44. Spencer, B.W., Jones, B.G.: Statistical investigation of pressure and velocity fields in the turbulent two-stream mixing layer. AIAA Paper 71, 613 (1971)

45. Speziale, C. G.: Analytical methods for the development of reynolds-stress closures in turbulence. Ann. Rev. Fluid Mech. 23, 107-157 (1991)

46. Stolz, S., Adams, N.: An approximate deconvolution procedure for large-eddy simulation. Phys. Fluids 11, 1699-1701 (1999)

47. Stolz, S., Adams, N. A., Kleiser, L.: An approximate deconvolution model applied for large-eddy simulation with application to incompressible wall-bounded flows. Phys. Fluids 13, 997 (2001)

48. Sutherland, J., Kennedy, C.: Improved boundary conditions for viscous, reacting, compressible flows. J. Comput. Phys. 191, 502-524 (2003)

49. Thacker, W., Sarkar, S., Gatski, T.: Analyzing the influence of compressibility on the rapid pressure-strain rate correlation in turbulent shear flows. Theor. Comput. Fluid Dyn. 21(3), 171-199 (2007)

50. Vreman, A. W., Sandham, N. D., Luo, K. H.: Compressible mixing layer growth rate and turbulence characteristics. J. Fluid Mech. 320, 235-258 (1996)

51. Vreman, B., Geurts, B., Kuerten, H.: Subgrid-modelling in LES of compressible flow. Appl. Sci. Res. 54, 191-203 (1995)

52. Vreman, B., Guerts, B., Kuerten, H.: Large-eddy simulation of the turbulent mixing layer. J. Fluid Mech. 339, 357-390 (1997)

53. Zhuang, M., Kubota, T., Dimotakis, P.: Instability of inviscid, compressible free shear layers. Phys. Fluids 28(10), $1728-1733(1991)$ 